University of Louisville

ThinkIR: The University of Louisville's Institutional Repository

Electronic Theses and Dissertations

1936

\title{
The effect of experimental liver damage on the blood picture of the dog.
}

H. D. Bruner

University of Louisville

Follow this and additional works at: https://ir.library.louisville.edu/etd

Part of the Anatomy Commons, Medical Sciences Commons, and the Pathological Conditions, Signs and Symptoms Commons

\section{Recommended Citation}

Bruner, H. D., "The effect of experimental liver damage on the blood picture of the dog." (1936). Electronic Theses and Dissertations. Paper 1701.

https://doi.org/10.18297/etd/1701

This Master's Thesis is brought to you for free and open access by ThinkIR: The University of Louisville's Institutional Repository. It has been accepted for inclusion in Electronic Theses and Dissertations by an authorized administrator of ThinkIR: The University of Louisville's Institutional Repository. This title appears here courtesy of the author, who has retained all other copyrights. For more information, please contact thinkir@louisville.edu. 
UNIVERSITY OF LOUISVILLE

\author{
THE EFFECT OF EXPERIMENTAL LIVER DAMAGE \\ ON THE BLOOD PICTURE OF THE DOG
}

\author{
A Dissertation \\ Submitted to the Faculty \\ Of the Graduate School of the University of Louisville \\ In Partial Fulfiliment of the \\ Requirements for the Degree \\ of Master of Science
}

Department of Phys10logy and Pharmacology

By

H. D. Bruner

1936 
3
3
5
+5
+5

CONTENTS

Page

INTRODUCTION . . . . . . . . . . . . . 1

METHOD . . . . . . . . . . . . . . . 4

RESULTS . . . . . . . . . . . . . . . II

DISCUSSION . . . . . . . . . . . . . 35

SUMMARY . . . . . . . . . . . . . . . 49

BIBLIOGRAPHY . . . . . . . . . . . . 52 


\section{ACKNOWLEDGEMENT}

To Dr. George E. Wakerlin, whose stimulating guidance and untiring efforts have made this work possible, I express my deepest appreciation. I wish to thank, also, Dr. John Walker Moore who made available the facilities of the Louisville City Hospital Laboratories, and Dr. A. J. Miller, under whose direction the sections were made. 


\section{INTRODUCTION}

Work during the past nine years suggests that various macrocytic hyperchromic anemias, of which pernicious anemia 1s an example, may result from: 1) the absence of a socalled intrinsic factor, normally contained in the gastric secretion $(1,2)$; 2) the absence of an extrinsic factor assoclated with the nitrogenous portion of the diet(3); 3) the lack of absorption $(4,5,6,7,8)$ of the product of the interaction $(2,9)$ of the intrinsic and extrinsic factors; 4) the interference with or derangement of the storage mechanism for this principle in the liver $(10,11,12,13,14,15)$. Acute or chronic liver damage as seen clinically may be accompanied by a typical macrocytic hyperchromic anemia presenting in a greater or lesser degree the cytological changes characteristic of pernicious anemia. Further, these patients respond promptly to treatment with liver preparations known to be effective in pernicious anemia. Wintrobe and Shumacker(16), reviewing forty-three cases of hepatic cirrhosis, note a $37 \%$ incidence of macrocytic hyperchromic anemia. Van Duyn(17) reports an $18 \%$ Incidence in twenty-oight cases of hepatic cirrhosis. Malamos (18) reports that in each of twenty-four cases of diffuse liver disease studied, the mean diameter of the erythrocytes was increased by $0.6-2.3 \mathrm{mlcra}$, together with a widening of the Price-Jones curve. 
Experimental corroboration of these clinical findings appears to be wanting. Salto(19), working chiefly with rabbits, found a distinct diminution in erythrocytes and hemoglobin upon excision of the liver, but the anemia produced bore no similarity to any of the macrocytic hyperchromic anemias. Bracalon 1 and Montanar1(20), having subtotally hepatectomized rabbits which subsequently died within five days due to hepatic insufficlency, found an increase in the number of the younger cells of the leukocytic serles and an increase in the nucleated and basophilic cells of the erythrocytic series in the circulating blood. They suggested that this observed blood picture was due to the removal of part of the reticulo-endothelial system. Pelligrini(21) reported that following total hepatectomy on frogs kept at a low temperature, there occurred an intense anemia; control operated animals showed a less marked but nevertheless definite anemia. He observed in the circulating blood a significant increase of the immature forms of the erythrocytic serles throughout the animals' subsequent life, which usually was 10 to 15 days with an extreme of 24 days. The bone marrow of the hepatectomized frogs showed an active proliferation of the erythrocytic elements accompanied by an arrest of the cyto-evolutive processes at the erythroblastic stage. Bollman and Mann(22,23) made occasional blood counts on their partialIy hepatectomized dogs and reported the presence of only a mild microcytic hypochromic anemia. About the time of 
completion of the work reported in this thesis, Higgins and Stasney(24) reported that an experimental cirrhosis of the liver together with a macrocytic anemia can be obtained in white rats by the inhalation of carbon tetrachloride vapor. The anemia produced is a macrocytic hypochromic anemia, thereby differing significantly from the type of anemia previously cited as associated with certain instances of hepatic cirrhoses in man. The carbon tetrachloride, moreover, affected organs other than the liver.

The foregoing facts suggested a study of the effect of chronic liver damage on the blood pleture of the dog. It was hoped that all or a part of the liver damaged animals would show evidences of a macrocytic hyperchromic anemia of such degree as to render them suitable for the bioassay of the antianemic activity of liver extracts. Such an animal would be useful in investigating both abnormal and normal hematopolesis. The liver of the dog appears to carry out a function in hematopoiesis similar to that of the liver of man. Ivy, Morgan and Farrel(25) and Strauss and Castle(26) have found that extracts of canine liver were effective in producing remission in cases of pernicious anemia, although the content of the ant1-pernicious anemia principle is one-fourth to onefifth that of hog liver.

Evidence that the dog may develop an anemia which is hematologically identical with the macrocytic hyperchromic anemias of the human is found in the reports of Stort1(27) 
and of Syderhelm(28). Stort 1 was able to induce the morphological picture of pernicious anemia in two of three dogs by infestation with Dibothriocephalus ranarum Gastalde. Syderhelm, by means of stenosis of the small intestine induced by operation, obtained a blood picture closely simulating that of pernicious anemia.

\section{METHOD:}

A search was made for a finely divided substance which upon injection into the portal circulation would produce interference with the capillary blood supply to the liver sufficlent to cause atrophy of the lobules of the liver with subsequent cirrhotic changes and diminished liver function. Finely divided carbon particles were found to vary too widely in size and to be quite irregularly shaped. Starch granules from various sources likewise varied too widely in diameter. Tiny glass spheres, had they been avallable, would have been most suitable. Lycopodium spores which are entirely inert physlologically, were found to have sultable diameters: between 38 to 112 micra with an average of $68 \mathrm{micra}$; they were sufficiently spherical to block more or less completely the flow of blood in any vessel of corresponding size in which they might lodge.

Preliminary experiments were performed for the purpose of: 1) determining the strength of suspension of spores which could be injected without clogging either the syringe, the 
needle, or the vein; 2) determining the maximum tolerated quantity of spores; 3) corroborating the findings of Mall (29) that material injected into the portal vein is evenly distributed to all parts of the liver.

From elght acute experiments on dogs under ether anesthesia, it was observed that the spores were most easily injected into the portal system and carried into the I1ver when an 18 gauge $1-1 / 2$ inch needle was inserted into a tertiary tributary of the superior mesenteric vein and pushed up inside the vein just past its junction with another to form a secondary tributary, the suspension then being injected at the rate of $10 \mathrm{cc}$. per minute. Upon withdrawal of the needle, the vein was ligatured at sites proximal and distal to the puncture. Among the various concentrations of spores, with and without various stabilizing agents, it was found that the most suitable was a one per cent suspension of spores in 0.9 per cent sodium chloride solution which, when autoclaved, remained stable during the time required for injection. Microscopic examination of frozen sections from various portions of the livers of these animals revealed a fairly even distribution of spores in the smallest intrahepatic portal radicles. A few spores were observed in the sinusoids and none in the central lobular veins. Doubtless many more spores were present in the sinusoids of these sections, but were rolled out by the microtome knife. Thereupon, a series of 17 dogs, 10 males and 7 females, 
were operated under sterile precautions, injecting the selected concentration of spores in various doses per k110gram. In the female series, it was found that $10 \mathrm{cc}$. per $\mathrm{kg}$. was fatal in two dogs and that doses of $7.5,7.5,7.5,5.0$, and $2.0 \mathrm{cc}$. per $\mathrm{kg}$. were tolerated. In the male serles, 7.5 cc. per $\mathrm{kg}$. was fatal in two dogs and doses of $2.5,2.5$, and $1.5 \mathrm{cc}$. per $\mathrm{kg}$. were also fatal; doses of $5.0,5.0,2.5$, 2.0 , and $1.5 \mathrm{cc}$. per $\mathrm{kg}$. were tolerated by other males. Therefore, dose of $7.5 \mathrm{cc}$. per $\mathrm{kg}$. for the females and $5.0 \mathrm{cc}$. per $\mathrm{kg}$. for the males was selected. At these levels in the final experimental series, there was a $43 \%$ mortality in seven females and $33 \%$ mortality in six males. With two exceptions, those animals which died did so within two hours after completion of the operation. In most instances they appeared to recover partially from the anesthesia and then to become unconscious. Very strong stimuli fa1led to elicit reflex activity. The tongue and mucous membranes became progressively paler. The respiration frequently took on cheyneStokes or Kussmaul characteristics. The heart beat became progressively weaker and more rapid. Rectal temperatures of 95 degrees F. frequently were recorded at the time of death. This type of death constitutes an acute form of the clinical syndrome observed in occlusive thrombophlebitis of the portal vein(30). Other workers have reported similar results upon experimental restriction of the blood flow in the portal vein $(31,32,33)$. 
Autopsy findings indicated a most severe obstruction to blood flow in the tissues drained by the portal vein. The gut from the distal third of the stomach to the middie of the colon was thickened, turgid and of a bluish-purple color; the mucosa was hemorrhagic and the lumen was filled with chocolate colored fluid. The spleen was three to five times larger than normal and oozed blood upon nicking the capsule. The liver was normal in size and consistancy but dark in color. The chambers of the heart contained but small amounts of blood. The lungs, kidneys, suprarenals, and the distal half of the colon were not remarkable. There was never found more than $50 \mathrm{cc}$. of fluid in the peritoneal cavity. All the autopsy findings, therefore, pointed to a damming back of the blood in the portal vein and its tributaries.

The final experimental series consisted of $s 1 x$ adult male and six adult, non-pregnant female dogs. These animals were entirely unselected except that they were objectively healthy. Throughout the experiment, the animals were housed Individually in roomy cages and fed on a standard balanced diet of canned dog food with an occasional bone. They obtained exercise while the cages were being cleaned and on their trips to the laboratory table. They were trained to Ile quietly on a table and submit to venepuncture and other handling without objective pain or fear.

During a four week control perlod, the erythrocytes, hemoglobin, hematocrit and leukocytes were determined at weekly 
intervals; the reticulocytes were determined semi-weekly; determinations of liver function by the Rose Bengal method and blood chemistry determinations were carried out on alternate weeks. Blood samples from the saphenous vein were drawn in the morning at approximately the same time for each dog in order to rule out diurnal variations as far as possible.

The blood for counting was drawn into a sterile, dry glass syringe containing heparin in quantities sufficient to make a concentration of one mgm. per $4 \mathrm{cc}$. of blood. At once the blood was thoroughly mixed with the anticoagulant and by means of a capillary pipette a part was placed in duplicate hematocrit tubes having a $3 \mathrm{~mm}$. bore. The remainder was spread in a thick lajer on an alcohol cleansed slide from which duplicate red blood cell, single hemoglobin, single white blood cell and single reticulocyte pipettes* were filled; thereupon, dilutions with appropriate fluids were quickly made. After homogeneous mixing with Hayem's fluid, duplicate erythrocyte determinations were made by filling each chamber of a bright line hemacytometer from separate pipettes and counting four hundred small squares of that chamber. The recorded figure was the average of two counts differing less than 200,000 . Leukocyte determinations were made by counting four square millimeters in each chamber filled from the same pipette and recording the average figure. Hemoglobin determinations were obtained by the Newcomer technique and instrument.

\footnotetext{
* Hemacytometer, red and white cell pipettes were certified
} by the United States Bureau of Standards. 
The hematocrit tubes were centrifuged at 2,200 revolutions per minute for forty minutes in a size 2 type $B$ International centrifuge with a working head of $13.5 \mathrm{~cm}$. radius. Preliminary work showed that at this speed complete packing usually had occurred at thirty minutes and always at thirty-five minutes. Readings were made to 0.25 per cent in the separate tubes and averaged. The top of the gray-red layer of white and red cells overlying the pure red-cell column was taken empirically as the line of demarcation. Thus the technique with certain insignificant adaptations follows that outlined by Wintrobe(34), who states(35) that the greatest variation in each of the constants, which can be attributed to the technique alone, can be represented by the mean coefficlents of variation plus three times their standard deviations, which in no instance exceeded $2.5 \%$. Th1s value may therefore be considered the maximum variation attributable to technique.

Using the wet-smear technique outlined by Stitt(36), the reticulocytes were recorded in per cent from a count of 1,000 cells of the erythrocytic series. The Rose Bengal function test $(37,38)$ was selected on the basis of accuracy, and simplicity. The technique described by Delprat and Stowe (37), was found to be applicable to the dogs; throughout, the dose of $2.06 \mathrm{mgm}$. Of dye per $\mathrm{kg}$. was used. Determinations of non-protein nitrogen, urea, creatinine, blood sugar, $\mathrm{CO}_{2} \mathrm{com-}$ bining power of plasma, and the van den Bergh test were made by the technical staff of the Louisville city Hospital 
Laboratories.

of the twelve animals, four males and four females were selected for the injection of lycopodium spores, the remaining four serving as operated control animals. Each animal was operated the day after the fourth count had been made. The dog, having been fasted for 24 hours, received a preoperative infection of $15 \mathrm{mgm}$. of morphine and $0.6 \mathrm{mgm}$. of atropine. Throughout the operation, which lasted an average of two hours, the animal was maintained in light surgical anesthesia by ether administered by the drop method. Under strict sterile precautions a high right rectus layered incision was made, the liver palpated and a biopsy of the Iiver obtained. The small intestine, preferably the ileum, was delivered into warm physiological saline sponges, a suitable vein selected, the spore suspension injected and the vein tied off. The liver was reinspected for evidence of bleeding from the blopsy area or from a possible mupture due to traction. The wound was then closed with silk in four layers. Control animals were subjected to identical manipulations except that 0.9 per cent sodium chloride solution in doses of 7.5 or $5.0 \mathrm{cc}$. per $\mathrm{kg}$. Instead of the spore suspension was injected into the portal circulation.

The blood studies were continued as during the preIIminary control period for nine weeks succeeding operation. Subsequently a clinically active preparation of liver extract* 
( 3 cc. derived from 100 grams of fresh Ilver) was injected into the hamstring muscles in doses of $0.2 \mathrm{cc}$. per $\mathrm{kg}$. daily for five days. Beginning with the third injection of liver extract, sem1-weekly samples of blood were obtained and cell counts made. Reticulocyte counts were made dally throughout this period. The alternating sequence of liver function and blood chemistry studies was not disturbed. The period of observation following the liver injections lasted four weeks.

At the end of this period, the animals with three exceptions were sacrificed and autopsled, and specimens of the livers were secured for microscopic examination.

\section{RESULTS:}

Data obtained by the described standardized technique from 63 counts on 19 normal dogs which were used in preliminary experiments are summarized in Table I and are submitted for the purpose of comparison with the data of the final experimental group of dogs, with special regard for the range of variation to be expected. The data obtained from the twelve animals of the final experimental group at weekly intervals during the preoperative control period are summarized in Table II. A comparison of Tables I and II indicates that the animals of the final experimental group were hematologically normal during the preoperative control period. In Table II are included the maximum variations noted in any single animal of the final experimental group between two successive sets of 
TABLE I

BLOOD VALUES FOR NORMAL DOGS

(63 counts on 19 animals)

\begin{tabular}{|c|c|c|c|}
\hline & Average & Maximum & Minimum \\
\hline $\begin{array}{l}\text { Erythrocytes } \\
\text { per crm. }\end{array}$ & $6,531,000$ & $8,545,000$ & $5,140,000$ \\
\hline $\begin{array}{l}\text { Hemoglobin } \\
\text { grs. per } 100 \mathrm{cc} \text {. }\end{array}$ & 13.82 & 18.1 & 10.4 \\
\hline $\begin{array}{l}\text { Hematocrit } \\
\text { per cent cells }\end{array}$ & 45.53 & 57.00 & 35.00 \\
\hline $\begin{array}{l}\text { Ret 1 culocytes } \\
\text { per cent } R \cdot B \cdot C \text {. }\end{array}$ & 0.35 & 1.1 & 0.0 \\
\hline $\begin{array}{l}\text { Leukocytes } \\
\text { per crmm. }\end{array}$ & 12,795 & 23,700 & 4,500 \\
\hline $\begin{array}{c}\text { Mean Corpuscular } \\
\text { Volume } \\
\text { (cuble micra) }\end{array}$ & 69.73 & 82.1 & 57.6 \\
\hline $\begin{array}{c}\text { Mean Corpuscular } \\
\text { Hemoglobin } \\
\text { (gamma gamma) }\end{array}$ & 21.43 & 26.0 & 17.6 \\
\hline $\begin{array}{l}\text { Mean Corpuscular } \\
\text { Hemoglobin Conc. } \\
\text { (per cent) }\end{array}$ & 30.75 & 34.3 & 27.9 \\
\hline
\end{tabular}


TABLE II

BLOOD VALUES OF DOGS USED IN EXPERIMENT DURING

PREOPERATIVE CONTROL PERIOD

\begin{tabular}{|c|c|c|c|c|}
\hline & Average & Maximum & Minimum & $\begin{array}{l}\text { Maximum } \\
\text { variation } \\
\text { between } 2 \\
\text { successive } \\
\text { counts }\end{array}$ \\
\hline $\begin{array}{l}\text { Erythrocytes } \\
\text { per cmm. }\end{array}$ & $6,647,000$ & $8,930,000$ & $5,330,000$ & $1,415,000$ \\
\hline $\begin{array}{l}\text { Hemoglobin } \\
\text { grs. per } 100 \mathrm{cc} .\end{array}$ & 14.29 & 18.64 & 11.38 & 2.6 \\
\hline $\begin{array}{c}\text { Hematocrit } \\
\text { per cent colls }\end{array}$ & 46.53 & 57.00 & 37.50 & 11.00 \\
\hline $\begin{array}{l}\text { Ret iculocytes } \\
\text { per cont R.B.C. }\end{array}$ & 0.46 & 2.3 & 0.0 & 1.9 \\
\hline $\begin{array}{l}\text { Leukocytes } \\
\text { per cmm. }\end{array}$ & 13,750 & 25,250 & 4,400 & 12,050 \\
\hline $\begin{array}{c}\text { Mean Corpuscular } \\
\text { Volume } \\
\text { (cuble micra) }\end{array}$ & 69.45 & 77.2 & 61.7 & 11.0 \\
\hline $\begin{array}{c}\text { Mean Corpus cular } \\
\text { Hemoglob1n } \\
\text { (gamma gamma) }\end{array}$ & 21.41 & 24.8 & 19.0 & 4.0 \\
\hline $\begin{array}{l}\text { Mean Corpuscular } \\
\text { Hemoglobin Conc. } \\
\text { (per cent) }\end{array}$ & 30.94 & 35.5 & 27.5 & 4.4 \\
\hline
\end{tabular}


observations. These variations are definitely beyond the limits of observational error. In Tables III to IX inclusive are shown in detall the erythrocyte counts, the hemoglobin determinations, the hematocrit determinations, the reticulocyte counts, the mean corpuscular volumes, the mean corpuscular hemoglobins, and the mean corpuscular hemoglobin concentrations for the twelve dogs during the preliminary control period of four weeks preceeding operation. These tables also show the same data for the dogs during the nine weeks succeding operation and during the four weeks following injections of liver extract. From these tables it is apparent that there were no significant changes in the erythrocyte counts, the hemoglobin determinations, the hematocrit determinations, the reticulocyte counts, the mean corpuscular volumes, the mean corpuscular hemoglobins, and the mean corpuscular hemoglobin concentrations in the animals of either the lycopodium spore injected group or the operated control group. It is also apparent from these tables that there were, likewise, no significant changes as the result of the liver extract injections. In other words all of the varlations observed were within the limits of normal(see Tables $I$ and II). From the data of Tables III to IX, Figures I and II were constructed using the arithmetical means for the two groups of dogs. Figures I and II show that there were no significant differences between the mean values of the erythrocytes, the hemoglobin determinations, the hematocrit 
TABLE III

ERYTEROCYTE COUNTS OF SPORE IIJECTED AND OPERATED CONTHOL DOGS in millions per cmm.

\begin{tabular}{|c|c|c|c|c|c|c|c|c|c|c|c|c|}
\hline \multirow{3}{*}{ Day } & \multicolumn{8}{|c|}{ Spore Injected } & \multicolumn{4}{|c|}{ Operatea Control } \\
\hline & \multicolumn{4}{|c|}{ Males } & \multicolumn{4}{|c|}{ Females } & \multicolumn{2}{|c|}{ Males } & \multicolumn{2}{|c|}{ Femeles } \\
\hline & $\# 2$ & $\# 4$ & $\# 6$ & $\# 8$ & $\# 1$ & \#9 & $t 10$ & $\# 12$ & 洋3 & 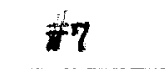 & $\# 5$ & $\# 11$ \\
\hline $\begin{array}{r}0 \\
7 \\
14 \\
21\end{array}$ & $\begin{array}{l}6.580 \\
7.100 \\
7.815 \\
6.715\end{array}$ & $\begin{array}{l}7.470 \\
6.250 \\
7.320 \\
6.640\end{array}$ & $\begin{array}{l}8.930 \\
8.330 \\
8.100 \\
8.320\end{array}$ & $\begin{array}{l}5.745 \\
6.230 \\
6.470 \\
5.700\end{array}$ & $\begin{array}{l}7.150 \\
5.735 \\
5.880 \\
6.240\end{array}$ & $\begin{array}{l}6.570 \\
6.550 \\
6.390 \\
6.580\end{array}$ & $\begin{array}{l}6.330 \\
6.860 \\
6.475 \\
5.980\end{array}$ & $\begin{array}{l}5.830 \\
6.445 \\
6.605 \\
6.020\end{array}$ & $\begin{array}{l}7.055 \\
6.800 \\
6.270 \\
5.575\end{array}$ & $\begin{array}{l}6.335 \\
5.330 \\
6.100 \\
6.280\end{array}$ & $\begin{array}{l}6.600 \\
7.700 \\
6.285 \\
6.335\end{array}$ & $\begin{array}{l}6.575 \\
6.430 \\
7.265 \\
7.715\end{array}$ \\
\hline 22 & \multicolumn{12}{|c|}{ Operation } \\
\hline $\begin{array}{l}28 \\
35 \\
42 \\
49 \\
56 \\
63 \\
70 \\
77 \\
84 \\
\end{array}$ & $\begin{array}{l}6.510 \\
6.470 \\
6.555 \\
6.015 \\
6.610 \\
6.285 \\
6.735 \\
7.380 \\
6.705 \\
\end{array}$ & $\begin{array}{l}7.340 \\
6.445 \\
6.880 \\
6.440 \\
6.575 \\
5.680 \\
6.685 \\
6.000 \\
7.120\end{array}$ & $\begin{array}{l}6.675 \\
7.145 \\
6.255 \\
7.440 \\
7.610 \\
7.080 \\
6.930 \\
7.570 \\
6.675 \\
\end{array}$ & $\begin{array}{l}5.300 \\
5.490 \\
6.170 \\
5.370 \\
5.275 \\
5.415 \\
4.880 \\
5.240 \\
5.445 \\
\end{array}$ & $\begin{array}{l}6.190 . \\
6.435 \\
6.290 \\
7.330 \\
6.840 \\
6.390 \\
8.470 \\
7.585 \\
7.980 \\
\end{array}$ & $\begin{array}{l}5.755 \\
6.355 \\
6.295 \\
6.000 \\
7.730 \\
7.700 \\
6.960 \\
6.465 \\
6.490 \\
\end{array}$ & $\begin{array}{l}7.320 \\
6.250 \\
6.665 \\
6.060 \\
6.165 \\
6.985 \\
6.790 \\
5.675 \\
7.110 \\
\end{array}$ & $\begin{array}{l}5.760 \\
6.035 \\
5.775 \\
5.600 \\
5.350 \\
5.985 \\
5.970 \\
5.600 \\
5.665 \\
\end{array}$ & $\begin{array}{l}6.135 \\
7.170 \\
6.310 \\
5.790 \\
6.385 \\
6.620 \\
5.300 \\
5.490 \\
5.805 \\
\end{array}$ & $\begin{array}{l}5.905 \\
5.905 \\
5.755 \\
6.190 \\
5.810 \\
5.605 \\
5.525 \\
5.480 \\
4.870\end{array}$ & $\begin{array}{l}5.945 \\
6.360 \\
5.930 \\
5.565 \\
5.755 \\
5.680 \\
6.315 \\
5.445 \\
5.850 \\
\end{array}$ & $\begin{array}{l}5.935 \\
6.970 \\
6.455 \\
6.935 \\
8.010 \\
6.590 \\
7.050 \\
6.930 \\
6.380 \\
\end{array}$ \\
\hline \multicolumn{13}{|c|}{ Inver Extract Injected 85th through 89th Deys } \\
\hline $\begin{array}{r}88 \\
91 \\
95 \\
98 \\
101 \\
105 \\
108 \\
112\end{array}$ & $\begin{array}{l}7.415 \\
6.725 \\
6.965 \\
6.740 \\
6.740 \\
6.575 \\
6.480 \\
6.965\end{array}$ & $\begin{array}{l}5.850 \\
6.325 \\
7.365 \\
6.390 \\
6.830 \\
6.015 \\
6.535 \\
7.140\end{array}$ & $\begin{array}{l}7.325 \\
6.955 \\
5.655 \\
6.780 \\
7.425 \\
6.875 \\
8.020 \\
7.205\end{array}$ & $\begin{array}{l}5.770 \\
5.355 \\
5.555 \\
5.530 \\
5.490 \\
5.775 \\
5.430 \\
5.230\end{array}$ & $\begin{array}{l}6.320 \\
7.370 \\
6.985 \\
7.690 \\
7.390 \\
7.895 \\
7.300 \\
7.910\end{array}$ & $\begin{array}{l}7.285 \\
7.290 \\
7.235 \\
7.545 \\
7.175 \\
8.785 \\
6.775 \\
5.990\end{array}$ & $\begin{array}{l}6.685 \\
6.430 \\
6.785 \\
6.240 \\
6.975 \\
6.890 \\
6.790 \\
6.980\end{array}$ & $\begin{array}{l}6.775 \\
6.190 \\
6.005 \\
6.120 \\
6.650 \\
6.680 \\
6.350 \\
6.535\end{array}$ & $\begin{array}{l}5.455 \\
6.400 \\
6.150 \\
5.605 \\
6.105 \\
5.765 \\
6.095 \\
6.055\end{array}$ & $\begin{array}{l}5.005 \\
4.945 \\
5.470 \\
5.125 \\
6.620 \\
5.010 \\
5.075 \\
5.410\end{array}$ & $\begin{array}{l}5.605 \\
6.445 \\
6.410 \\
6.510 \\
6.465 \\
6.780 \\
7.065 \\
7.100\end{array}$ & $\begin{array}{l}6.770 \\
6.715 \\
7.150 \\
6.670 \\
6.815 \\
6.650 \\
7.300 \\
6.955\end{array}$ \\
\hline
\end{tabular}


TABLE IV

HEMOGLOBIN VALUES OF' SPORE INJECTED AND OPERATED CONTROL DOGS

in grams per $100 \mathrm{cc}$. of blood (Newcomer scale)

\begin{tabular}{|c|c|c|c|c|c|c|c|c|c|c|c|c|}
\hline \multirow{3}{*}{ Day } & \multicolumn{8}{|c|}{ Spore Injected } & \multicolumn{4}{|c|}{ Operated Control } \\
\hline & \multicolumn{4}{|c|}{ rales } & \multicolumn{4}{|c|}{ Females } & \multicolumn{2}{|c|}{ Males } & \multicolumn{2}{|c|}{ Females } \\
\hline & Hz & $\$ 4$ & 46 & it8 & $\# 1$ & $\# 9$ & $\neq 10$ & $\$ 12$ & \#3 & $\# 7$ & A5 & $\# 11$ \\
\hline $\begin{array}{r}0 \\
7 \\
14 \\
21\end{array}$ & $\begin{array}{l}16.3 \\
14.8 \\
15.5 \\
15.4\end{array}$ & $\begin{array}{l}15.1 \\
13.1 \\
15.7 \\
15.5\end{array}$ & $\begin{array}{l}18.6 \\
16.6 \\
17.2 \\
16.5\end{array}$ & $\begin{array}{l}11.8 \\
13.5 \\
12.9 \\
12.1\end{array}$ & $\begin{array}{l}13.6 \\
11.5 \\
11.4 \\
12.6\end{array}$ & $\begin{array}{l}13.0 \\
14.5 \\
13.1 \\
14.0\end{array}$ & $\begin{array}{l}14.9 \\
15.0 \\
15.1 \\
12.8\end{array}$ & $\begin{array}{l}14.0 \\
13.7 \\
14.0 \\
13.7\end{array}$ & $\begin{array}{l}16.9 \\
16.2 \\
13.9 \\
16.2\end{array}$ & $\begin{array}{l}13.4 \\
13.2 \\
13.4 \\
13.7\end{array}$ & $\begin{array}{l}14.0 \\
14.3 \\
13.3 \\
13.7\end{array}$ & $\begin{array}{l}13.4 \\
14.3 \\
15.4 \\
15.0\end{array}$ \\
\hline 22 & \multicolumn{12}{|c|}{ Operation } \\
\hline $\begin{array}{l}28 \\
35 \\
42 \\
49 \\
56 \\
63 \\
70 \\
77 \\
84\end{array}$ & $\begin{array}{l}14.1 \\
13.3 \\
13.7 \\
13.3 \\
13.8 \\
12.7 \\
14.1 \\
15.2 \\
13.8\end{array}$ & $\begin{array}{l}15.0 \\
14.2 \\
14.1 \\
13.1 \\
13.4 \\
13.4 \\
14.0 \\
12.9 \\
14.1\end{array}$ & $\begin{array}{l}14.7 \\
13.3 \\
13.1 \\
13.9 \\
13.8 \\
14.2 \\
13.6 \\
15.0 \\
14.0\end{array}$ & $\begin{array}{r}12.1 \\
11.7 \\
12.3 \\
11.0 \\
11.7 \\
9.8 \\
9.9 \\
10.2 \\
11.5\end{array}$ & $\begin{array}{l}13.1 \\
12.8 \\
14.1 \\
13.7 \\
14.0 \\
13.9 \\
15.5 \\
15.3 \\
15.8\end{array}$ & $\begin{array}{l}13.3 \\
12.4 \\
14.8 \\
13.0 \\
14.4 \\
14.1 \\
14.0 \\
14.2 \\
14.5\end{array}$ & $\begin{array}{l}14.8 \\
14.4 \\
14.3 \\
14.0 \\
14.5 \\
15.2 \\
14.5 \\
12.1 \\
14.8\end{array}$ & $\begin{array}{l}12.9 \\
13.7 \\
12.0 \\
12.1 \\
11.1 \\
12.1 \\
13.5 \\
11.5 \\
11.6\end{array}$ & $\begin{array}{l}15.1 \\
15.7 \\
14.2 \\
13.7 \\
14.1 \\
14.1 \\
13.7 \\
12.6 \\
12.5\end{array}$ & $\begin{array}{r}14.1 \\
12.6 \\
12.3 \\
12.1 \\
11.5 \\
10.7 \\
11.6 \\
10.1 \\
9.9\end{array}$ & $\begin{array}{l}11.9 \\
12.7 \\
12.1 \\
12.0 \\
11.7 \\
12.0 \\
11.9 \\
11.7 \\
11.9\end{array}$ & $\begin{array}{l}13.7 \\
14.8 \\
13.2 \\
14.2 \\
15.2 \\
14.2 \\
14.7 \\
14.2 \\
13.8\end{array}$ \\
\hline \multicolumn{13}{|c|}{ Iiver Extract } \\
\hline $\begin{array}{r}88 \\
91 \\
95 \\
98 \\
101 \\
105 \\
108 \\
112\end{array}$ & $\begin{array}{l}15.4 \\
15.3 \\
14.2 \\
13.2 \\
14.0 \\
14.0 \\
14.7 \\
13.9\end{array}$ & $\begin{array}{l}13.3 \\
14.3 \\
13.8 \\
13.2 \\
14.0 \\
13.4 \\
14.0 \\
14.1\end{array}$ & $\begin{array}{l}14.2 \\
13.3 \\
11.6 \\
14.5 \\
14.7 \\
14.1 \\
15.5 \\
14.6\end{array}$ & $\begin{array}{l}10.7 \\
10.8 \\
11.6 \\
11.6 \\
11.3 \\
11.2 \\
12.0 \\
11.3\end{array}$ & $\begin{array}{l}13.9 \\
15.1 \\
15.0 \\
15.2 \\
16.0 \\
17.0 \\
16.0 \\
15.3\end{array}$ & $\begin{array}{l}15.7 \\
14.8 \\
13.5 \\
11.6 \\
15.4 \\
15.0 \\
14.3 \\
13.8\end{array}$ & $\begin{array}{l}14.1 \\
14.6 \\
14.2 \\
15.2 \\
14.6 \\
14.0 \\
15.1 \\
15.1\end{array}$ & $\begin{array}{l}13.6 \\
13.3 \\
12.9 \\
14.6 \\
13.8 \\
13.6 \\
13.0 \\
13.3\end{array}$ & $\begin{array}{l}12.1 \\
13.6 \\
12.5 \\
10.3 \\
12.4 \\
12.1 \\
12.9 \\
12.3\end{array}$ & $\begin{array}{r}10.2 \\
9.7 \\
9.6 \\
10.6 \\
9.8 \\
9.8 \\
9.6 \\
9.9\end{array}$ & $\begin{array}{l}10.3 \\
12.2 \\
11.8 \\
12.0 \\
13.0 \\
12.3 \\
13.0 \\
13.3\end{array}$ & $\begin{array}{l}13.6 \\
13.9 \\
14.3 \\
13.8 \\
12.8 \\
13.3 \\
14.4 \\
14.3\end{array}$ \\
\hline
\end{tabular}


TABLE V

HEMATOCRIT DEPERMINATIONS OF SPORE INJECTED AND OPERATED CONTROL DOGS in per cent cells

\begin{tabular}{|c|c|c|c|c|c|c|c|c|c|c|c|c|}
\hline \multirow{3}{*}{ Day } & \multicolumn{8}{|c|}{ Spore Injected } & \multirow{2}{*}{\multicolumn{4}{|c|}{\begin{tabular}{l|l} 
Operated Control \\
Males & Females
\end{tabular}}} \\
\hline & \multicolumn{4}{|c|}{ Males } & \multicolumn{4}{|c|}{ Females } & & & & \\
\hline & $\# 2$ & $7_{4}$ & $\frac{3}{4} 6$ & $\$ 8$ & 71 & $\# 9$ & $\# 10$ & $\# 12$ & \pm 3 & $A \eta$ & $\# 5$ & $\# 11$ \\
\hline $\begin{array}{r}0 \\
7 \\
14 \\
21\end{array}$ & $\begin{array}{l}49.50 \\
45.00 \\
49.75 \\
46.75\end{array}$ & $\begin{array}{l}48.25 \\
43.00 \\
54.00 \\
48.00\end{array}$ & $\begin{array}{l}57.00 \\
53.50 \\
55.00 \\
52.25\end{array}$ & $\begin{array}{l}41.50 \\
43.50 \\
44.25 \\
42.25\end{array}$ & $\begin{array}{l}46.25 \\
37.50 \\
39.75 \\
42.50\end{array}$ & $\begin{array}{l}45.25 \\
48.75 \\
45.00 \\
46.50\end{array}$ & $\begin{array}{l}49.75 \\
50.00 \\
44.00 \\
41.00\end{array}$ & $\begin{array}{l}45.00 \\
46.00 \\
40.75 \\
43.75\end{array}$ & $\begin{array}{l}54.00 \\
52.50 \\
45.25 \\
50.50\end{array}$ & $\begin{array}{l}43.00 \\
41.00 \\
42.00 \\
43.75\end{array}$ & $\begin{array}{l}45.50 \\
48.00 \\
42.50 \\
42.00\end{array}$ & $\begin{array}{l}42.75 \\
47.00 \\
48.50 \\
49.00\end{array}$ \\
\hline 22 & \multicolumn{12}{|c|}{ Operation } \\
\hline $\begin{array}{l}28 \\
35 \\
42 \\
49 \\
56 \\
63 \\
70 \\
77 \\
84\end{array}$ & $\begin{array}{l}45.00 \\
45.00 \\
46.75 \\
44.00 \\
43.75 \\
41.25 \\
46.50 \\
48.75 \\
45.25\end{array}$ & $\begin{array}{l}48.25 \\
45.75 \\
45.50 \\
42.50 \\
43.00 \\
44.75 \\
44.75 \\
42.50 \\
47.25\end{array}$ & $\begin{array}{l}45.25 \\
45.75 \\
46.25 \\
43.00 \\
45.00 \\
46.25 \\
43.75 \\
49.75 \\
46.25\end{array}$ & $\begin{array}{l}36.75 \\
38.00 \\
42.00 \\
38.25 \\
39.75 \\
35.00 \\
34.50 \\
38.75 \\
40.00\end{array}$ & $\begin{array}{l}41.75 \\
43.75 \\
47.25 \\
48.00 \\
46.00 \\
44.75 \\
54.00 \\
51.50 \\
54.25\end{array}$ & $\begin{array}{l}42.25 \\
41.25 \\
49.50 \\
44.00 \\
47.75 \\
47.75 \\
46.50 \\
45.25 \\
47.25\end{array}$ & $\begin{array}{l}49.75 \\
41.50 \\
46.75 \\
46.50 \\
46.50 \\
49.25 \\
48.75 \\
42.75 \\
47.00\end{array}$ & $\begin{array}{l}40.50 \\
44.00 \\
39.75 \\
39.00 \\
37.75 \\
42.50 \\
43.75 \\
39.75 \\
39.75\end{array}$ & $\begin{array}{l}46.50 \\
48.00 \\
45.75 \\
45.00 \\
44.25 \\
44.50 \\
44.25 \\
40.50 \\
41.00\end{array}$ & $\begin{array}{l}41.50 \\
41.50 \\
41.00 \\
39.00 \\
39.75 \\
36.25 \\
38.75 \\
36.00 \\
31.50\end{array}$ & $\begin{array}{l}38.25 \\
41.75 \\
39.50 \\
39.25 \\
39.25 \\
38.75 \\
40.50 \\
39.50 \\
39.25\end{array}$ & $\begin{array}{l}42.25 \\
43.25 \\
42.50 \\
44.00 \\
48.75 \\
44.00 \\
47.00 \\
45.75 \\
42.00\end{array}$ \\
\hline \multicolumn{13}{|c|}{ Iiver Extract Injected 85th through 89th Days } \\
\hline $\begin{array}{r}88 \\
91 \\
95 \\
98 \\
101 \\
105 \\
108 \\
112\end{array}$ & $\begin{array}{l}49.25 \\
48.25 \\
46.75 \\
43.75 \\
45.50 \\
44.75 \\
48.00 \\
46.00\end{array}$ & $\begin{array}{l}43.00 \\
44.25 \\
47.00 \\
45.25 \\
45.75 \\
43.50 \\
46.50 \\
46.50\end{array}$ & $\begin{array}{l}46.25 \\
44.25 \\
39.75 \\
47.75 \\
47.50 \\
47.50 \\
51.50 \\
48.50\end{array}$ & $\begin{array}{l}38.75 \\
39.00 \\
40.00 \\
39.75 \\
40.00 \\
40.00 \\
40.25 \\
38.00\end{array}$ & $\begin{array}{l}46.00 \\
51.50 \\
51.00 \\
54.75 \\
50.75 \\
53.25 \\
50.00 \\
49.00\end{array}$ & $\begin{array}{l}48.50 \\
47.25 \\
46.25 \\
49.00 \\
51.25 \\
48.50 \\
48.00 \\
41.50\end{array}$ & $\begin{array}{l}45.00 \\
46.50 \\
47.00 \\
48.50 \\
49.50 \\
46.50 \\
50.25 \\
48.50\end{array}$ & $\begin{array}{l}45.25 \\
42.50 \\
41.25 \\
42.75 \\
44.75 \\
45.50 \\
44.50 \\
43.75\end{array}$ & $\begin{array}{l}39.50 \\
42.00 \\
40.00 \\
38.00 \\
41.00 \\
40.00 \\
42.25 \\
41.75\end{array}$ & $\begin{array}{l}33.25 \\
41.00 \\
33.00 \\
34.75 \\
37.00 \\
34.00 \\
34.50 \\
35.50\end{array}$ & $\begin{array}{l}34.50 \\
32.50 \\
37.75 \\
41.00 \\
43.00 \\
43.00 \\
41.25 \\
42.25\end{array}$ & $\begin{array}{l}44.00 \\
43.00 \\
45.00 \\
45.00 \\
42.50 \\
43.00 \\
48.75 \\
47.00\end{array}$ \\
\hline
\end{tabular}


PABIE VI

RETICULOCYTE COUNTS OF SPORE INJECTED AND OPERATED CONTROL DOGS in per cent

\begin{tabular}{|c|c|c|c|c|c|c|c|c|c|c|c|c|}
\hline \multirow{3}{*}{ Day } & \multicolumn{8}{|c|}{ Spore Injected } & \multicolumn{4}{|c|}{ Operated Control } \\
\hline & \multicolumn{4}{|c|}{ Males } & \multicolumn{4}{|c|}{ Females } & \multicolumn{2}{|c|}{ Nales } & \multicolumn{2}{|c|}{ Females } \\
\hline & $\frac{y}{72}$ & $\# 4$ & it6 & $\neq 8$ & 荤 & t49 & \#10 & $\# 12$ & $\# 3$ & $\# 7$ & $\frac{n}{75}$ & $\# 11$ \\
\hline $\begin{array}{r}0 \\
3 \\
7 \\
10 \\
14 \\
17 \\
21\end{array}$ & $\begin{array}{l}0.0 \\
0.1 \\
0.7 \\
0.0 \\
0.7 \\
0.5 \\
0.2\end{array}$ & $\begin{array}{l}0.6 \\
0.3 \\
0.1 \\
1.0 \\
0.8 \\
0.3 \\
0.5\end{array}$ & $\begin{array}{l}0.2 \\
0.1 \\
0.2 \\
0.0 \\
0.0 \\
0.0 \\
0.0\end{array}$ & $\begin{array}{l}1.2 \\
1.8 \\
1.8 \\
2.3 \\
1.2 \\
0.4 \\
1.2\end{array}$ & $\begin{array}{l}1.4 \\
1.5 \\
0.4 \\
1.7 \\
1.1 \\
1.6 \\
0.9\end{array}$ & $\begin{array}{l}0.0 \\
0.0 \\
0.6 \\
0.2 \\
0.5 \\
0.6 \\
1.2\end{array}$ & $\begin{array}{l}0.0 \\
0.4 \\
0.7 \\
0.6 \\
0.4 \\
0.5 \\
0.7\end{array}$ & $\begin{array}{l}0.5 \\
0.3 \\
0.5 \\
0.1 \\
0.1 \\
0.1 \\
0.1\end{array}$ & $\begin{array}{l}0.2 \\
0.1 \\
0.3 \\
0.2 \\
0.2 \\
0.4 \\
0.4\end{array}$ & $\begin{array}{l}0.1 \\
0.8 \\
0.0 \\
0.3 \\
0.4 \\
0.3 \\
0.0\end{array}$ & $\begin{array}{l}0.0 \\
0.1 \\
0.0 \\
0.0 \\
0.0 \\
0.2 \\
0.4\end{array}$ & $\begin{array}{l}0.2 \\
0.6 \\
0.3 \\
0.4 \\
0.2 \\
0.1 \\
0.2\end{array}$ \\
\hline 22 & \multicolumn{12}{|c|}{ Operation } \\
\hline $\begin{array}{l}24 \\
28 \\
31 \\
35 \\
38 \\
42 \\
45 \\
49 \\
52 \\
56 \\
59 \\
63 \\
66 \\
70 \\
73 \\
77 \\
80 \\
84\end{array}$ & $\begin{array}{l}0.7 \\
0.3 \\
0.1 \\
0.0 \\
0.2 \\
0.3 \\
0.0 \\
0.4 \\
0.0 \\
0.1 \\
0.2 \\
0.2 \\
0.4 \\
0.3 \\
0.3 \\
0.4 \\
0.4 \\
0.1\end{array}$ & $\begin{array}{l}0.4 \\
0.4 \\
0.3 \\
0.9 \\
0.1 \\
0.3 \\
0.1 \\
1.1 \\
1.1 \\
1.2 \\
0.8 \\
1.0 \\
0.8 \\
1.3 \\
0.4 \\
1.1 \\
0.7 \\
0.8\end{array}$ & $\begin{array}{l}0.2 \\
0.0 \\
0.0 \\
0.0 \\
0.0 \\
0.4 \\
0.0 \\
0.1 \\
0.3 \\
0.5 \\
0.0 \\
0.2 \\
0.3 \\
0.3 \\
0.1 \\
0.1 \\
0.0 \\
0.0\end{array}$ & $\begin{array}{l}0.3 \\
0.0 \\
0.4 \\
0.9 \\
1.2 \\
0.9 \\
0.1 \\
1.1 \\
0.8 \\
1.0 \\
0.6 \\
0.8 \\
0.4 \\
1.2 \\
1.3 \\
1.8 \\
1.2 \\
0.8\end{array}$ & $\begin{array}{l}0.4 \\
0.4 \\
0.4 \\
1.1 \\
0.8 \\
1.1 \\
0.4 \\
0.8 \\
0.6 \\
1.0 \\
0.6 \\
0.8 \\
1.0 \\
1.2 \\
0.4 \\
1.0 \\
0.7 \\
0.8\end{array}$ & $\begin{array}{l}0.3 \\
0.5 \\
0.3 \\
0.1 \\
0.7 \\
0.7 \\
0.7 \\
0.7 \\
0.7 \\
0.4 \\
0.0 \\
0.5 \\
0.0 \\
0.3 \\
0.3 \\
0.4 \\
0.4 \\
1.0\end{array}$ & $\begin{array}{l}0.5 \\
0.6 \\
0.8 \\
0.3 \\
0.3 \\
0.4 \\
0.0 \\
0.1 \\
0.2 \\
0.1 \\
0.1 \\
0.1 \\
0.1 \\
0.2 \\
0.2 \\
0.1 \\
0.0 \\
0.0\end{array}$ & $\begin{array}{l}0.2 \\
0.1 \\
0.0 \\
0.2 \\
0.0 \\
0.2 \\
0.0 \\
0.3 \\
0.7 \\
0.7 \\
0.8 \\
0.1 \\
0.5 \\
0.3 \\
0.5 \\
0.3 \\
0.1 \\
0.0\end{array}$ & $\begin{array}{l}0.1 \\
0.4 \\
0.0 \\
0.2 \\
0.1 \\
0.1 \\
0.3 \\
0.3 \\
0.0 \\
0.3 \\
0.1 \\
0.1 \\
0.0 \\
0.2 \\
0.1 \\
0.5 \\
0.5 \\
0.2\end{array}$ & $\begin{array}{l}0.5 \\
0.4 \\
0.3 \\
0.7 \\
0.4 \\
0.0 \\
0.4 \\
1.4 \\
0.2 \\
0.7 \\
0.7 \\
1.2 \\
0.5 \\
1.3 \\
0.5 \\
1.6 \\
0.6 \\
1.0\end{array}$ & $\begin{array}{l}0.8 \\
0.0 \\
0.8 \\
1.9 \\
1.3 \\
0.7 \\
0.3 \\
1.5 \\
0.2 \\
1.2 \\
0.2 \\
0.3 \\
0.1 \\
0.4 \\
0.5 \\
0.6 \\
0.2 \\
0.2\end{array}$ & $\begin{array}{l}0.2 \\
0.2 \\
0.5 \\
0.4 \\
0.1 \\
0.2 \\
0.0 \\
0.1 \\
0.1 \\
0.2 \\
0.0 \\
0.0 \\
0.1 \\
0.5 \\
0.7 \\
0.8 \\
0.2 \\
0.1\end{array}$ \\
\hline
\end{tabular}


TABLE VI (Concluded)

\begin{tabular}{|c|c|c|c|c|c|c|c|c|c|c|c|c|}
\hline \multirow{3}{*}{ Day } & \multicolumn{8}{|c|}{ Spore Injected } & \multicolumn{4}{|c|}{ Operated Control } \\
\hline & \multicolumn{4}{|c|}{ Males } & \multicolumn{4}{|c|}{ Females } & \multicolumn{2}{|c|}{ MaIes } & \multicolumn{2}{|c|}{ Females } \\
\hline & $\# 2$ & \#4 & \#6 & \#8 & \#1 & \#9 & $\# 10$ & \#12 & $\# 3$ & $\# 7$ & \#5 & \#11 \\
\hline \multicolumn{13}{|c|}{ Iiver Extract Injected 85th through } \\
\hline $\begin{array}{r}85 \\
86 \\
87 \\
88 \\
89 \\
90 \\
91 \\
92 \\
93 \\
94 \\
95 \\
96 \\
97 \\
98 \\
99 \\
100 \\
101 \\
102 \\
103 \\
104 \\
105 \\
106 \\
107 \\
108 \\
109 \\
110 \\
111 \\
112\end{array}$ & $\begin{array}{l}0.3 \\
0.2 \\
0.4 \\
0.2 \\
0.5 \\
0.2 \\
0.5 \\
0.3 \\
0.0 \\
0.3 \\
0.1 \\
0.1 \\
0.5 \\
0.2 \\
0.2 \\
0.0 \\
0.1 \\
0.0 \\
0.0 \\
0.1 \\
0.3 \\
0.0 \\
0.0 \\
0.0 \\
0.0 \\
0.1 \\
0.0 \\
0.2\end{array}$ & $\begin{array}{l}0.9 \\
0.8 \\
0.7 \\
1.3 \\
0.8 \\
1.6 \\
1.1 \\
1.4 \\
1.3 \\
1.5 \\
0.8 \\
1.1 \\
0.9 \\
0.2 \\
1.2 \\
0.6 \\
0.7 \\
1.0 \\
1.7 \\
0.5 \\
0.8 \\
0.9 \\
0.3 \\
1.0 \\
1.0 \\
0.7 \\
1.7 \\
1.2\end{array}$ & $\begin{array}{l}0.1 \\
0.0 \\
0.2 \\
0.1 \\
0.3 \\
0.4 \\
0.4 \\
1.1 \\
0.5 \\
0.0 \\
0.2 \\
0.0 \\
0.2 \\
0.0 \\
0.1 \\
0.1 \\
0.1 \\
0.1 \\
0.0 \\
0.0 \\
0.0 \\
0.1 \\
0.1 \\
0.1 \\
0.1 \\
0.0 \\
0.3 \\
0.2\end{array}$ & $\begin{array}{l}0.6 \\
0.8 \\
0.5 \\
1.2 \\
0.6 \\
0.8 \\
1.2 \\
1.1 \\
1.9 \\
1.2 \\
0.7 \\
0.3 \\
0.7 \\
1.0 \\
0.5 \\
0.4 \\
0.3 \\
0.4 \\
0.6 \\
0.9 \\
1.4 \\
1.2 \\
0.4 \\
2.8 \\
1.5 \\
1.2 \\
1.8 \\
1.4\end{array}$ & $\begin{array}{l}0.5 \\
0.7 \\
0.8 \\
0.5 \\
1.0 \\
0.1 \\
0.3 \\
0.3 \\
0.3 \\
0.1 \\
0.4 \\
0.2 \\
0.1 \\
0.5 \\
0.4 \\
0.3 \\
0.1 \\
0.5 \\
0.3 \\
0.1 \\
0.6 \\
0.0 \\
0.6 \\
0.2 \\
0.2 \\
0.1 \\
0.0 \\
0.3\end{array}$ & $\begin{array}{l}0.2 \\
0.1 \\
0.3 \\
0.0 \\
0.3 \\
0.0 \\
0.3 \\
0.0 \\
0.1 \\
0.1 \\
0.0 \\
0.1 \\
0.2 \\
0.2 \\
0.1 \\
0.2 \\
0.1 \\
0.2 \\
0.1 \\
0.0 \\
0.4 \\
0.3 \\
0.1 \\
0.4 \\
0.4 \\
0.0 \\
0.3 \\
0.1\end{array}$ & $\begin{array}{l}0.0 \\
0.0 \\
0.0 \\
0.1 \\
0.0 \\
0.1 \\
0.2 \\
0.2 \\
0.4 \\
0.0 \\
0.3 \\
0.3 \\
0.0 \\
0.1 \\
0.1 \\
0.0 \\
0.0 \\
0.1 \\
0.1 \\
0.1 \\
0.4 \\
0.2 \\
0.3 \\
0.2 \\
0.3 \\
0.2 \\
0.0 \\
0.1\end{array}$ & $\begin{array}{l}0.7 \\
0.4 \\
0.2 \\
0.2 \\
0.7 \\
0.4 \\
0.2 \\
0.2 \\
0.0 \\
0.1 \\
0.5 \\
0.0 \\
0.1 \\
0.1 \\
0.6 \\
0.2 \\
0.4 \\
0.4 \\
0.2 \\
0.2 \\
0.3 \\
0.4 \\
0.4 \\
0.8 \\
0.2 \\
0.4 \\
0.2 \\
0.3\end{array}$ & $\begin{array}{l}0.2 \\
0.1 \\
0.2 \\
0.3 \\
0.0 \\
0.2 \\
0.2 \\
0.2 \\
0.2 \\
0.0 \\
0.1 \\
0.2 \\
0.0 \\
0.0 \\
0.2 \\
0.0 \\
0.1 \\
0.2 \\
0.4 \\
0.1 \\
0.2 \\
0.4 \\
0.0 \\
0.1 \\
0.2 \\
0.0 \\
0.2 \\
0.2\end{array}$ & $\begin{array}{l}0.8 \\
0.5 \\
0.8 \\
1.5 \\
1.7 \\
0.8 \\
1.5 \\
0.4 \\
1.9 \\
1.4 \\
0.4 \\
1.1 \\
0.2 \\
2.1 \\
1.2 \\
1.5 \\
1.0 \\
0.8 \\
0.6 \\
0.4 \\
1.0 \\
0.7 \\
1.2 \\
0.8 \\
0.2 \\
0.4 \\
0.2 \\
0.5\end{array}$ & $\begin{array}{l}0.2 \\
0.4 \\
0.1 \\
0.0 \\
0.1 \\
0.4 \\
0.1 \\
0.0 \\
0.0 \\
0.1 \\
0.6 \\
0.3 \\
0.1 \\
0.4 \\
0.0 \\
0.0 \\
0.2 \\
0.2 \\
0.0 \\
0.1 \\
0.1 \\
0.1 \\
0.2 \\
0.4 \\
0.4 \\
0.3 \\
0.0 \\
0.2\end{array}$ & $\begin{array}{l}0.2 \\
0.3 \\
0.1 \\
0.1 \\
0.1 \\
0.2 \\
0.1 \\
0.4 \\
0.1 \\
0.4 \\
0.1 \\
0.1 \\
0.4 \\
0.1 \\
0.6 \\
0.4 \\
0.6 \\
0.3 \\
0.1 \\
0.6 \\
0.3 \\
0.1 \\
0.1 \\
0.0 \\
0.4 \\
0.2 \\
0.1 \\
0.4\end{array}$ \\
\hline
\end{tabular}


TABIE VII

MFAN CORPUSCULAR VOLUMES OF SPORE INJECTED AND OPERATED COINTROL DOGS in cubic micra

\begin{tabular}{|c|c|c|c|c|c|c|c|c|c|c|c|c|}
\hline \multirow{3}{*}{ Day } & \multicolumn{8}{|c|}{ Spore Injected } & \multicolumn{4}{|c|}{ Operated Control } \\
\hline & \multicolumn{4}{|c|}{ Males } & \multicolumn{4}{|c|}{ Females } & \multicolumn{2}{|c|}{ Miales } & \multicolumn{2}{|c|}{ Females } \\
\hline & $\frac{A 2}{A 2}$ & $\# 4$ & 76 & $\$ 8$ & \#1 & \#9 & +10 & $\# 12$ & $\$ 3$ & 87 & $\$ 5$ & $\# 11$ \\
\hline $\begin{array}{r}0 \\
7 \\
14 \\
21\end{array}$ & $\begin{array}{l}73.7 \\
63.4 \\
63.7 \\
69.6\end{array}$ & $\begin{array}{l}64.6 \\
68.8 \\
73.8 \\
72.3\end{array}$ & $\begin{array}{l}63.8 \\
64.2 \\
67.9 \\
62.8\end{array}$ & $\begin{array}{l}72.2 \\
69.8 \\
68.4 \\
74.1\end{array}$ & $\begin{array}{l}64.7 \\
65.4 \\
67.6 \\
69.6\end{array}$ & $\begin{array}{l}68.9 \\
74.4 \\
70.4 \\
70.7\end{array}$ & $\begin{array}{l}72.8 \\
72.8 \\
69.5 \\
68.6\end{array}$ & $\begin{array}{l}77.2 \\
69.2 \\
61.7 \\
72.7\end{array}$ & $\begin{array}{l}76.6 \\
77.2 \\
72.1 \\
75.1\end{array}$ & $\begin{array}{l}67.9 \\
76.9 \\
68.9 \\
69.7\end{array}$ & $\begin{array}{l}68.9 \\
68.6 \\
67.6 \\
66.3\end{array}$ & $\begin{array}{l}65.0 \\
73.1 \\
66.8 \\
63.5\end{array}$ \\
\hline 22 & \multicolumn{12}{|c|}{ Operation } \\
\hline $\begin{array}{l}28 \\
35 \\
42 \\
49 \\
56 \\
63 \\
70 \\
77 \\
84\end{array}$ & $\begin{array}{l}69.1 \\
69.6 \\
71.2 \\
73.2 \\
66.0 \\
65.6 \\
69.2 \\
64.7 \\
67.5\end{array}$ & $\begin{array}{l}65.7 \\
71.0 \\
66.1 \\
68.0 \\
65.4 \\
78.8 \\
66.9 \\
70.8 \\
65.0\end{array}$ & $\begin{array}{l}67.8 \\
64.0 \\
73.9 \\
57.8 \\
59.1 \\
65.3 \\
63.1 \\
65.7 \\
69.3\end{array}$ & $\begin{array}{l}69.4 \\
70.1 \\
68.1 \\
71.2 \\
75.7 \\
64.6 \\
70.7 \\
73.5 \\
73.5\end{array}$ & $\begin{array}{l}67.5 \\
68.0 \\
75.1 \\
65.5 \\
67.2 \\
70.0 \\
65.8 \\
67.9 \\
67.9\end{array}$ & $\begin{array}{l}73.4 \\
64.9 \\
78.6 \\
73.3 \\
67.0 \\
62.0 \\
66.8 \\
65.4 \\
72.8\end{array}$ & $\begin{array}{l}68.0 \\
66.4 \\
70.1 \\
76.6 \\
75.4 \\
70.5 \\
71.8 \\
75.3 \\
66.1\end{array}$ & $\begin{array}{l}70.3 \\
72.9 \\
68.8 \\
69.6 \\
70.6 \\
71.0 \\
73.3 \\
71.0 \\
70.3\end{array}$ & $\begin{array}{l}75.8 \\
64.2 \\
72.5 \\
77.7 \\
69.3 \\
67.2 \\
70.2 \\
72.0 \\
70.6\end{array}$ & $\begin{array}{l}70.3 \\
70.3 \\
71.0 \\
63.0 \\
68.4 \\
64.7 \\
70.1 \\
65.7 \\
64.7\end{array}$ & $\begin{array}{l}64.3 \\
65.6 \\
66.6 \\
70.5 \\
68.2 \\
68.2 \\
63.4 \\
72.5 \\
67.1\end{array}$ & $\begin{array}{l}71.2 \\
62.1 \\
65.8 \\
63.4 \\
60.9 \\
66.8 \\
66.7 \\
66.0 \\
65.8\end{array}$ \\
\hline \multicolumn{13}{|c|}{ Iiver Extract Injected 85th through 89 th Days } \\
\hline $\begin{array}{r}88 \\
91 \\
95 \\
98 \\
101 \\
105 \\
108 \\
112\end{array}$ & $\begin{array}{l}66.4 \\
71.8 \\
67.2 \\
64.9 \\
67.4 \\
68.2 \\
74.1 \\
66.0\end{array}$ & $\begin{array}{l}73.5 \\
70.0 \\
63.8 \\
65.3 \\
67.0 \\
72.3 \\
71.2 \\
65.1\end{array}$ & $\begin{array}{l}63.3 \\
63.6 \\
70.3 \\
70.0 \\
65.6 \\
69.0 \\
64.2 \\
67.3\end{array}$ & $\begin{array}{l}71.0 \\
70.5 \\
72.0 \\
71.9 \\
72.9 \\
72.3 \\
72.3 \\
72.7\end{array}$ & $\begin{array}{l}72.8 \\
69.9 \\
73.0 \\
71.1 \\
62.7 \\
67.6 \\
68.5 \\
61.9\end{array}$ & $\begin{array}{l}66.7 \\
64.8 \\
63.9 \\
64.9 \\
72.3 \\
59.2 \\
70.9 \\
69.3\end{array}$ & $\begin{array}{l}67.3 \\
72.2 \\
69.4 \\
77.7 \\
71.0 \\
67.5 \\
74.0 \\
69.5\end{array}$ & $\begin{array}{l}66.8 \\
68.7 \\
68.7 \\
69.8 \\
67.3 \\
68.1 \\
70.1 \\
66.2\end{array}$ & $\begin{array}{l}72.4 \\
65.6 \\
64.7 \\
67.8 \\
65.8 \\
69.4 \\
69.3 \\
69.0\end{array}$ & $\begin{array}{l}66.5 \\
65.7 \\
60.3 \\
67.8 \\
65.9 \\
67.9 \\
68.0 \\
65.6\end{array}$ & $\begin{array}{l}61.7 \\
63.6 \\
58.7 \\
61.6 \\
66.5 \\
63.4 \\
58.4 \\
59.5\end{array}$ & $\begin{array}{l}65.0 \\
64.0 \\
64.3 \\
67.5 \\
62.4 \\
64.7 \\
66.8 \\
67.6\end{array}$ \\
\hline
\end{tabular}


TABIE VIII

MEAN CORPUSCUTAR HEMOGLOBINS OF SPORE INJECTED AND OPERATED CONTROT DOGS in gamma gamma

\begin{tabular}{|c|c|c|c|c|c|c|c|c|c|c|c|c|}
\hline \multirow{3}{*}{ Day } & \multicolumn{8}{|c|}{ Spore Injected } & \multicolumn{4}{|c|}{ Operated Control } \\
\hline & \multicolumn{4}{|c|}{ Males } & \multicolumn{4}{|c|}{ Females } & \multicolumn{2}{|c|}{ 16les } & \multicolumn{2}{|c|}{ Females } \\
\hline & +2 & $\# 4$ & $\# 6$ & \#8 & H1 & $\neq 9$ & $\# 10$ & 是12 & 43 & $\# 7$ & 75 & 㩆1 \\
\hline $\begin{array}{r}0 \\
7 \\
14 \\
21 \\
\end{array}$ & $\begin{array}{l}24.8 \\
20.8 \\
19.7 \\
21.7\end{array}$ & $\begin{array}{l}20.2 \\
21.0 \\
21.3 \\
23.3\end{array}$ & $\begin{array}{l}20.9 \\
19.9 \\
21.2 \\
19.8\end{array}$ & $\begin{array}{l}19.8 \\
21.6 \\
19.9 \\
21.2\end{array}$ & $\begin{array}{l}19.0 \\
20.0 \\
19.3 \\
20.2 \\
\end{array}$ & $\begin{array}{l}19.9 \\
22.1 \\
20.5 \\
21.3 \\
\end{array}$ & $\begin{array}{l}21.8 \\
21.8 \\
23.3 \\
21.0\end{array}$ & $\begin{array}{l}24.0 \\
20.6 \\
21.1 \\
22.8\end{array}$ & $\begin{array}{l}24.0 \\
23.8 \\
22.2 \\
24.6\end{array}$ & $\begin{array}{l}21.1 \\
24.8 \\
21.9 \\
21.8 \\
\end{array}$ & $\begin{array}{l}21.2 \\
20.4 \\
21.2 \\
21.6\end{array}$ & $\begin{array}{l}20.4 \\
22.4 \\
21.2 \\
19.4\end{array}$ \\
\hline 22 & \multicolumn{12}{|c|}{ Operation } \\
\hline $\begin{array}{l}28 \\
35 \\
42 \\
49 \\
56 \\
63 \\
70 \\
77 \\
84\end{array}$ & $\begin{array}{l}21.7 \\
20.6 \\
20.9 \\
22.2 \\
20.9 \\
20.2 \\
21.0 \\
20.6 \\
20.6\end{array}$ & $\begin{array}{l}20.4 \\
22.1 \\
20.5 \\
20.4 \\
20.4 \\
23.6 \\
20.9 \\
21.6 \\
19.7\end{array}$ & $\begin{array}{l}22.0 \\
18.6 \\
21.0 \\
18.6 \\
18.1 \\
20.1 \\
19.1 \\
21.0 \\
19.8\end{array}$ & $\begin{array}{l}22.9 \\
21.3 \\
19.9 \\
20.5 \\
22.2 \\
18.0 \\
20.3 \\
19.3 \\
21.0\end{array}$ & $\begin{array}{l}21.2 \\
19.8 \\
21.4 \\
18.7 \\
20.5 \\
21.7 \\
18.3 \\
20.2 \\
19.8\end{array}$ & $\begin{array}{l}21.1 \\
19.5 \\
23.5 \\
21.6 \\
20.2 \\
18.3 \\
20.1 \\
20.4 \\
22.4\end{array}$ & $\begin{array}{l}20.2 \\
23.0 \\
21.5 \\
23.1 \\
23.6 \\
21.8 \\
21.3 \\
21.3 \\
20.8\end{array}$ & $\begin{array}{l}22.5 \\
22.7 \\
20.8 \\
21.6 \\
20.7 \\
20.2 \\
22.6 \\
20.6 \\
20.5\end{array}$ & $\begin{array}{l}24.8 \\
21.9 \\
20.9 \\
23.6 \\
22.1 \\
21.3 \\
21.7 \\
23.0 \\
21.5\end{array}$ & $\begin{array}{l}23.7 \\
21.3 \\
21.4 \\
19.5 \\
19.9 \\
19.0 \\
21.0 \\
18.5 \\
20.3\end{array}$ & $\begin{array}{l}20.1 \\
20.0 \\
20.5 \\
21.6 \\
20.4 \\
21.1 \\
18.9 \\
21.5 \\
20.3\end{array}$ & $\begin{array}{l}23.1 \\
21.3 \\
20.4 \\
20.5 \\
19.0 \\
21.4 \\
20.8 \\
20.6 \\
21.6\end{array}$ \\
\hline \multicolumn{13}{|c|}{ Liver Extract Injected 85th through 89 th Days } \\
\hline $\begin{array}{r}88 \\
91 \\
95 \\
98 \\
101 \\
105 \\
108 \\
112\end{array}$ & $\begin{array}{l}20.7 \\
22.8 \\
20.3 \\
19.6 \\
20.6 \\
21.2 \\
22.6 \\
20.0\end{array}$ & $\begin{array}{l}22.8 \\
22.5 \\
18.7 \\
19.0 \\
20.5 \\
22.3 \\
21.4 \\
19.7\end{array}$ & $\begin{array}{l}19.4 \\
19.1 \\
20.5 \\
21.4 \\
20.4 \\
20.4 \\
19.3 \\
20.3\end{array}$ & $\begin{array}{l}19.5 \\
19.5 \\
20.9 \\
20.9 \\
20.6 \\
21.7 \\
22.1 \\
21.7\end{array}$ & $\begin{array}{l}22.0 \\
20.5 \\
21.5 \\
19.7 \\
20.1 \\
21.4 \\
21.9 \\
19.3\end{array}$ & $\begin{array}{l}21.5 \\
19.9 \\
18.7 \\
19.3 \\
21.7 \\
18.3 \\
21.3 \\
22.4\end{array}$ & $\begin{array}{l}21.0 \\
22.7 \\
20.9 \\
25.0 \\
20.9 \\
20.3 \\
22.2 \\
21.7\end{array}$ & $\begin{array}{l}20.0 \\
21.4 \\
21.5 \\
20.9 \\
20.7 \\
20.4 \\
20.4 \\
20.4\end{array}$ & $\begin{array}{l}22.2 \\
21.3 \\
20.2 \\
18.4 \\
20.3 \\
21.1 \\
21.1 \\
20.3\end{array}$ & $\begin{array}{l}20.3 \\
19.7 \\
17.6 \\
20.7 \\
19.6 \\
19.5 \\
18.9 \\
18.3\end{array}$ & $\begin{array}{l}18.4 \\
19.0 \\
18.4 \\
18.5 \\
19.0 \\
19.1 \\
18.4 \\
18.7\end{array}$ & $\begin{array}{l}20.0 \\
20.7 \\
20.0 \\
20.7 \\
19.5 \\
19.3 \\
19.7 \\
20.6\end{array}$ \\
\hline
\end{tabular}


PABIE IX

MEAN CORPUSCULAR WHOGLOBIN CONCHTERATIONS OF SPORE INJECTED AND OPERATED CONTROL DOGS in per cent

\begin{tabular}{|c|c|c|c|c|c|c|c|c|c|c|c|c|}
\hline \multirow{3}{*}{ Day } & \multicolumn{8}{|c|}{ Spore Injected } & \multicolumn{4}{|c|}{ Operated Control } \\
\hline & \multicolumn{4}{|c|}{ Males } & \multicolumn{4}{|c|}{ Females } & \multicolumn{2}{|c|}{ Meles } & \multicolumn{2}{|c|}{ Females } \\
\hline & $\$ 2$ & $\# 4$ & 76 & $\frac{H}{178}$ & 71 & $\# 9$ & $\$ 10$ & $\$ 12$ & +3 & $\$ 7$ & $\# 5$ & $\# 1$ \\
\hline $\begin{array}{r}0 \\
7 \\
14 \\
21\end{array}$ & $\begin{array}{l}33.0 \\
32.8 \\
31.3 \\
31.1\end{array}$ & $\begin{array}{l}31.3 \\
30.5 \\
29.1 \\
32.3\end{array}$ & $\begin{array}{l}33.1 \\
31.0 \\
31.3 \\
31.5\end{array}$ & $\begin{array}{l}27.5 \\
30.9 \\
29.9 \\
28.6\end{array}$ & $\begin{array}{l}29.4 \\
30.6 \\
28.6 \\
29.7\end{array}$ & $\begin{array}{l}28.8 \\
29.7 \\
29.2 \\
30.1\end{array}$ & $\begin{array}{l}29.8 \\
29.9 \\
34.3 \\
31.2\end{array}$ & $\begin{array}{l}31.1 \\
29.8 \\
34.2 \\
31.3\end{array}$ & $\begin{array}{l}31.3 \\
30.9 \\
30.8 \\
32.1\end{array}$ & $\begin{array}{l}31.1 \\
29.8 \\
31.8 \\
31.2\end{array}$ & $\begin{array}{l}30.7 \\
32.2 \\
31.4 \\
32.5\end{array}$ & $\begin{array}{l}31.4 \\
30.5 \\
31.8 \\
30.6\end{array}$ \\
\hline 22 & \multicolumn{12}{|c|}{ Operation } \\
\hline $\begin{array}{l}28 \\
35 \\
42 \\
49 \\
56 \\
63 \\
70 \\
77 \\
84\end{array}$ & $\begin{array}{l}31.3 \\
29.6 \\
29.3 \\
30.3 \\
31.6 \\
30.7 \\
30.4 \\
31.1 \\
30.4\end{array}$ & $\begin{array}{l}31.0 \\
31.1 \\
31.0 \\
30.9 \\
31.2 \\
30.0 \\
31.3 \\
30.4 \\
29.7\end{array}$ & $\begin{array}{l}32.4 \\
29.1 \\
28.4 \\
32.2 \\
30.5 \\
30.7 \\
31.0 \\
30.3 \\
30.3\end{array}$ & $\begin{array}{l}33.0 \\
30.4 \\
29.3 \\
28.8 \\
29.4 \\
27.9 \\
28.8 \\
26.2 \\
28.7\end{array}$ & $\begin{array}{l}31.7 \\
29.1 \\
29.8 \\
28.5 \\
30.5 \\
31.0 \\
28.6 \\
29.6 \\
29.1\end{array}$ & $\begin{array}{l}31.5 \\
30.0 \\
29.9 \\
29.4 \\
30.2 \\
29.5 \\
30.1 \\
31.2 \\
31.0\end{array}$ & $\begin{array}{l}29.8 \\
34.7 \\
30.7 \\
30.1 \\
31.2 \\
30.9 \\
29.7 \\
28.3 \\
31.4\end{array}$ & $\begin{array}{l}32.0 \\
31.1 \\
30.2 \\
31.0 \\
29.3 \\
28.5 \\
30.9 \\
29.0 \\
28.9\end{array}$ & $\begin{array}{l}32.4 \\
34.2 \\
31.0 \\
30.4 \\
31.8 \\
31.6 \\
30.9 \\
31.1 \\
30.4\end{array}$ & $\begin{array}{l}34.0 \\
30.3 \\
30.1 \\
31.0 \\
29.0 \\
29.4 \\
30.0 \\
28.2 \\
31.4\end{array}$ & $\begin{array}{l}32.5 \\
30.5 \\
30.7 \\
30.6 \\
29.9 \\
30.9 \\
29.5 \\
29.7 \\
30.3\end{array}$ & $\begin{array}{l}32.5 \\
34.3 \\
31.0 \\
32.3 \\
31.2 \\
31.8 \\
31.3 \\
31.1 \\
32.8\end{array}$ \\
\hline & & & & Iive & xtract & Inject & a. $85 t$ & throus & $89 t h \mathrm{I}$ & & & \\
\hline $\begin{array}{r}88 \\
91 \\
95 \\
98 \\
101 \\
105 \\
108 \\
112\end{array}$ & $\begin{array}{l}31.2 \\
31.8 \\
30.3 \\
30.2 \\
30.7 \\
31.2 \\
30.8 \\
30.2\end{array}$ & $\begin{array}{l}31.0 \\
32.3 \\
29.4 \\
29.1 \\
30.6 \\
30.9 \\
30.0 \\
30.2\end{array}$ & $\begin{array}{l}30.7 \\
30.1 \\
28.9 \\
30.4 \\
31.0 \\
29.6 \\
30.0 \\
30.2\end{array}$ & $\begin{array}{l}27.6 \\
28.4 \\
28.9 \\
29.1 \\
28.2 \\
28.1 \\
29.9 \\
29.6\end{array}$ & $\begin{array}{l}30.2 \\
29.4 \\
29.4 \\
27.7 \\
31.4 \\
31.8 \\
31.9 \\
30.8\end{array}$ & $\begin{array}{l}32.3 \\
30.6 \\
29.1 \\
29.8 \\
30.0 \\
30.9 \\
29.8 \\
32.3\end{array}$ & $\begin{array}{l}31.2 \\
31.4 \\
30.1 \\
32.4 \\
29.5 \\
30.1 \\
30.1 \\
31.2\end{array}$ & $\begin{array}{l}30.0 \\
31.2 \\
31.3 \\
30.0 \\
30.7 \\
29.9 \\
29.1 \\
30.8\end{array}$ & $\begin{array}{l}30.6 \\
32.4 \\
31.1 \\
27.1 \\
30.2 \\
30.3 \\
30.5 \\
29.4\end{array}$ & $\begin{array}{l}30.6 \\
29.9 \\
29.2 \\
30.5 \\
29.5 \\
28.7 \\
27.8 \\
27.8\end{array}$ & $\begin{array}{l}29.8 \\
29.8 \\
31.8 \\
29.3 \\
28.6 \\
30.2 \\
31.5 \\
31.4\end{array}$ & $\begin{array}{l}30.8 \\
32.3 \\
31.7 \\
30.7 \\
31.3 \\
29.9 \\
29.5 \\
30.4\end{array}$ \\
\hline
\end{tabular}




\section{FIGURE I}

AVERAGE ERYTHROCYTE COUNTS, HEMOGLOBIN VALUES, HEMATOCRIT DETERM INATIONS, AND RETICULOCYTE COUNTS OF EIGHT SPORE INJECTED AND

FOUR OPERATED CONTROL DOGS

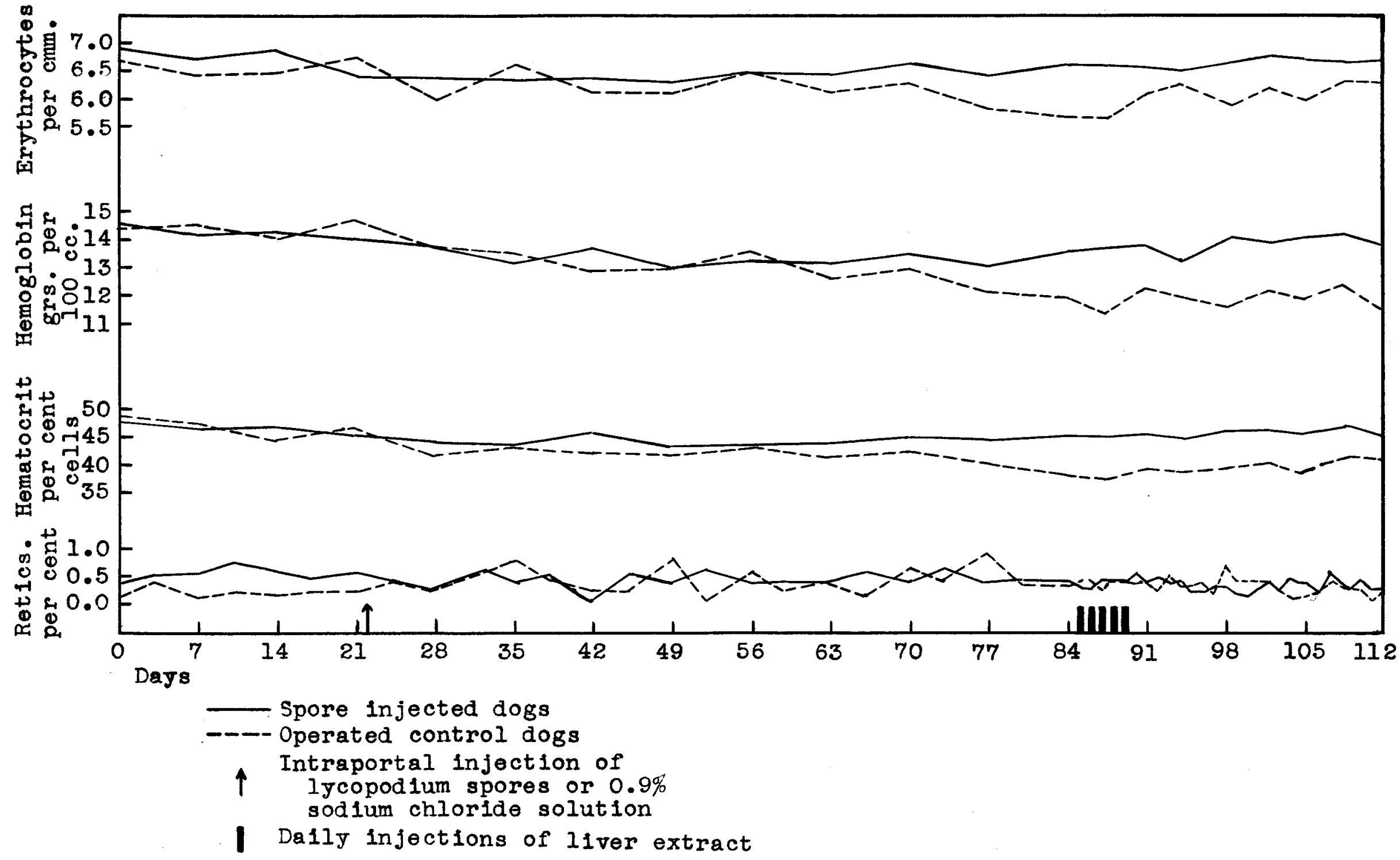


AVERAGE MEAN CORPUSCULAR VOLUMES, MEAN CORPUSCULAR HEMOGLOBINS,

AND MEAN CORPUSCULAR HEMOGLOBIN CONCENTRATIONS OF EIGHT

SPORE INJECTED AND FOUR OPERATED CONTROL DOGS

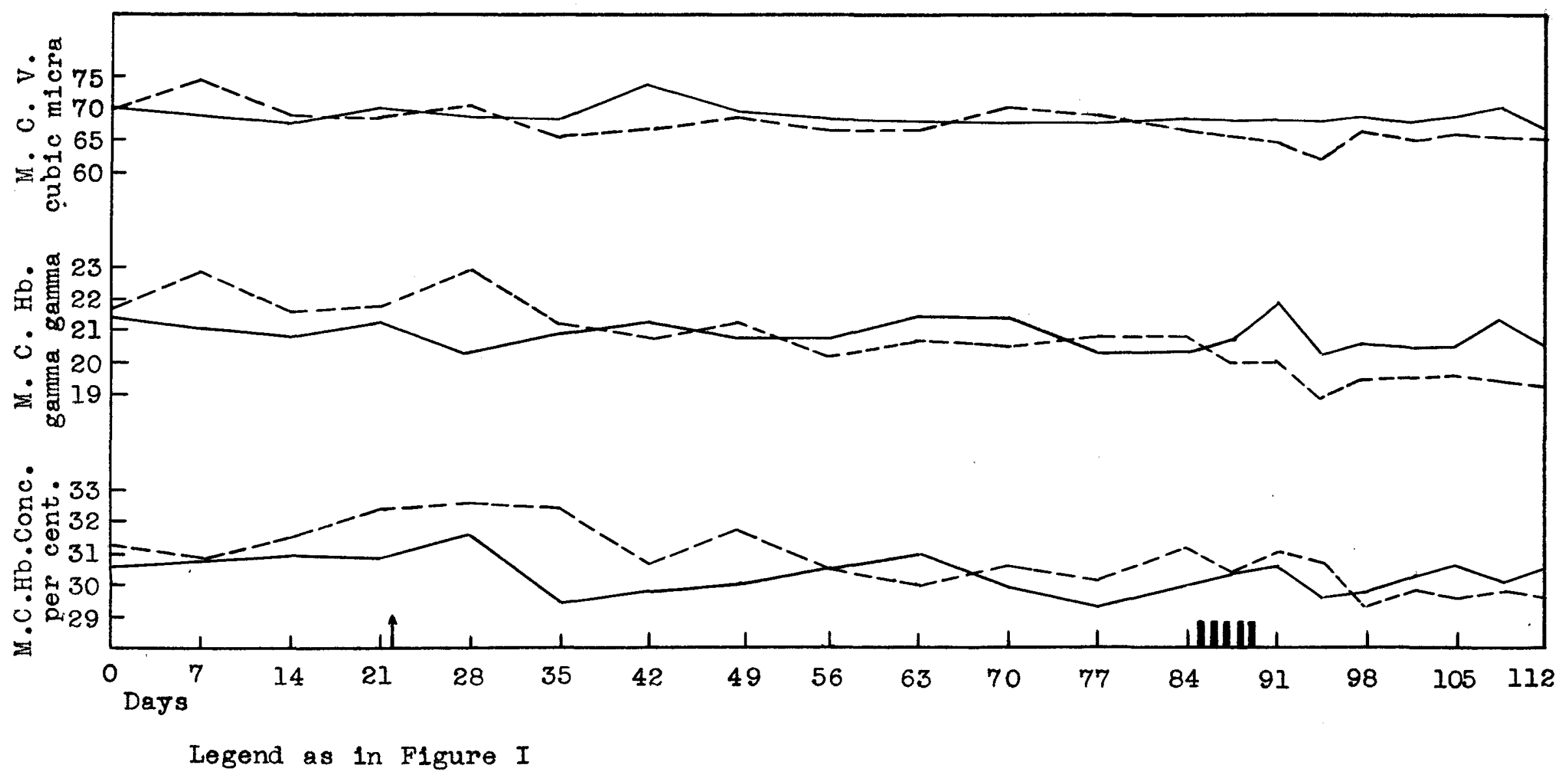


determinations, the reticulocyte counts, the mean corpuscular volumes, the mean corpuscular hemoglobins, and the mean corpuscular hemoglobin concentrations of the operated control and spore injected groups of dogs during the four weeks preIiminary control period. Similar curves (not included in this thesis) for the individual animals also show that there were no significant differences between data of the two groups of dogs. Figures I and II demonstrate further that during the nine weeks subsequent to operation there were no significant differences between the mean values of the data for the spore injected and operated control groups of animals. After the injection of IIver extract there were likewise no significant differences between the mean values of the two groups of anfmals.

Table $X$, consisting of the average erythrocyte counts and average mean corpuscular volumes of the twelve dogs during the preliminary control period, indicates a certain amount of correlation between the erythrocyte count and the mean corpuscular volume for most of the dogs. In particular, dogs \#2, \#6, and \#11 have the highest average erythrocyte counts and the lowest average mean corpuscular volumes. On the other hand, dogs \#l, and \#3 fail to show evidence of such a correlation. These findings are evident also from Figure III. Blood films made on the day of the last count for each dog were negative for Bartonella canis $(39,40)$.

In Table XI are shown the leukocyte counts for the 
TABLE $X$

AVERAGE ERYTHROCYTE COUNTS AND MEAN CORPUSCULAR VOLUMES OF THE SPORE INJECTED AND OPERATED CONTROL DOGS DURING THE FOUR WEEKS PREOPERATIVE CONTROL PERIOD

\begin{tabular}{|c|c|c|}
\hline Dog & $\begin{array}{c}\text { Erythrocytes } \\
\text { mililons } \\
\text { per cmm. }\end{array}$ & $\begin{array}{c}\text { Mean Corpuscular } \\
\text { Volume } \\
\text { cubic micra }\end{array}$ \\
\hline$\# 6$ & 8.4 & 64.7 \\
$\# 2$ & 7.1 & 67.3 \\
$\# 11$ & 7.0 & 67.1 \\
$\# 4$ & 6.9 & 69.9 \\
$\# 5$ & 6.7 & 75.3 \\
$\# 10$ & 6.6 & 67.9 \\
$\# 9$ & 6.5 & 70.9 \\
$\# 12$ & 6.5 & 71.1 \\
$\# 1$ & 6.2 & 70.2 \\
$\# 8$ & 6.2 & 66.8 \\
$\# 7$ & 6.0 & 71.1 \\
\hline
\end{tabular}




\section{FIGURE III}

RELATION OF AVERAGE ERYTHROCYTE COUNTS TO

MEAN CORPUSCILAR VOLUMES DURING THE

PREOPERATIVE CONTROL PERIOD

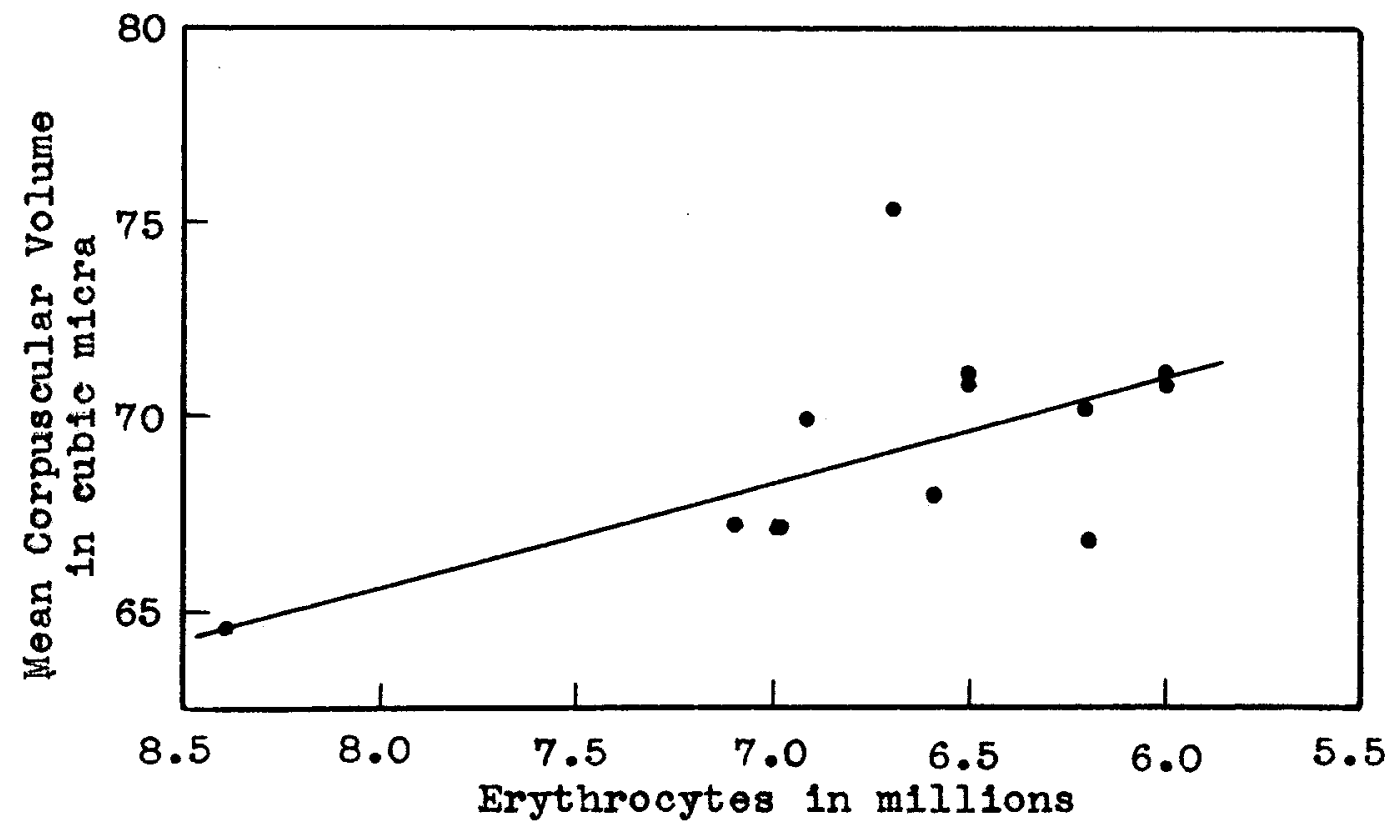




\section{TABIE XI}

LEUKOGYTE COUNTS OF SPORE INJECFED AND OPERATHD CONTROI DOGS in thousands per cmm.

\begin{tabular}{|c|c|c|c|c|c|c|c|c|c|c|c|c|}
\hline \multirow{3}{*}{ Day } & \multicolumn{8}{|c|}{ Spore Injected } & \multicolumn{4}{|c|}{ Operated Control } \\
\hline & \multicolumn{4}{|c|}{ Males } & \multicolumn{4}{|c|}{ Females } & \multicolumn{2}{|c|}{ Hales } & \multicolumn{2}{|c|}{ Females } \\
\hline & \#2 & 74 & m6 & 48 & 㳻 & \#9 & to & 㭗工2 & 43 & $\#$ & 45 & 11 \\
\hline $\begin{array}{r}0 \\
7 \\
14 \\
21\end{array}$ & $\begin{array}{l}13.35 \\
14.55 \\
12.80 \\
11.00\end{array}$ & $\begin{array}{l}18.45 \\
17.50 \\
15.35 \\
15.10\end{array}$ & $\begin{array}{r}4.60 \\
8.65 \\
10.95 \\
15.50\end{array}$ & $\begin{array}{r}8.75 \\
18.30 \\
25.25 \\
23.75\end{array}$ & $\begin{array}{r}18.50 \\
11.80 \\
23.85 \\
9.40\end{array}$ & $\begin{array}{r}17.30 \\
8.85 \\
12.40 \\
10.50\end{array}$ & $\begin{array}{r}11.70 \\
12.50 \\
4.40 \\
8.30\end{array}$ & $\begin{array}{l}19.95 \\
17.60 \\
13.75 \\
18.90\end{array}$ & $\begin{array}{r}9.55 \\
13.35 \\
11.75 \\
11.00\end{array}$ & $\begin{array}{l}15.45 \\
24.90 \\
17.75 \\
12.75\end{array}$ & $\begin{array}{r}4.50 \\
9.95 \\
12.50 \\
8.65\end{array}$ & $\begin{array}{r}16.75 \\
13.40 \\
14.75 \\
9.55\end{array}$ \\
\hline 22 & \multicolumn{12}{|c|}{ Operation } \\
\hline $\begin{array}{l}28 \\
35 \\
42 \\
49 \\
56 \\
63 \\
70 \\
77 \\
84\end{array}$ & $\begin{array}{r}8.30 \\
9.00 \\
10.60 \\
15.20 \\
8.85 \\
12.20 \\
12.35 \\
15.15 \\
14.40\end{array}$ & $\begin{array}{r}14.10 \\
11.90 \\
12.90 \\
11.10 \\
12.40 \\
10.20 \\
6.35 \\
14.40 \\
13.80\end{array}$ & $\begin{array}{r}8.60 \\
4.80 \\
11.85 \\
10.40 \\
10.25 \\
7.30 \\
12.75 \\
19.55 \\
8.15\end{array}$ & $\begin{array}{l}13.55 \\
27.30 \\
30.05 \\
19.70 \\
17.60 \\
28.85 \\
22.60 \\
24.50 \\
20.90 \\
\end{array}$ & $\begin{array}{l}17.10 \\
25.65 \\
19.95 \\
17.70 \\
15.35 \\
13.85 \\
13.35 \\
11.55 \\
17.30\end{array}$ & $\begin{array}{r}10.15 \\
18.10 \\
15.20 \\
21.60 \\
13.95 \\
17.10 \\
12.95 \\
8.60 \\
7.25 \\
\end{array}$ & $\begin{array}{r}10.50 \\
10.10 \\
5.75 \\
10.70 \\
8.05 \\
9.40 \\
9.65 \\
7.65 \\
10.55 \\
\end{array}$ & $\begin{array}{r}11.15 \\
19.65 \\
12.50 \\
15.20 \\
15.00 \\
17.30 \\
12.50 \\
15.20 \\
9.35 \\
\end{array}$ & $\begin{array}{r}8.50 \\
6.80 \\
14.40 \\
9.05 \\
6.30 \\
7.75 \\
10.00 \\
11.95 \\
10.85 \\
\end{array}$ & $\begin{array}{r}17.50 \\
14.40 \\
7.75 \\
11.90 \\
11.55 \\
13.15 \\
14.35 \\
14.90 \\
21.10 \\
\end{array}$ & $\begin{array}{r}7.10 \\
10.90 \\
12.80 \\
12.15 \\
12.35 \\
11.95 \\
11.40 \\
10.75 \\
12.90\end{array}$ & $\begin{array}{r}9.40 \\
7.35 \\
15.55 \\
12.10 \\
10.25 \\
13.85 \\
14.85 \\
12.20 \\
16.60 \\
\end{array}$ \\
\hline \multicolumn{13}{|c|}{ Iiver Extract Injected 85th through 89 th Days } \\
\hline $\begin{array}{r}88 \\
91 \\
95 \\
98 \\
101 \\
105 \\
108 \\
112\end{array}$ & $\begin{array}{r}19.55 \\
13.20 \\
20.90 \\
17.50 \\
14.20 \\
16.60 \\
13.50 \\
6.45\end{array}$ & $\begin{array}{r}18.25 \\
18.50 \\
11.85 \\
15.95 \\
17.95 \\
12.20 \\
13.70 \\
8.55\end{array}$ & $\begin{array}{r}22.40 \\
10.65 \\
9.35 \\
11.00 \\
6.50 \\
12.40 \\
17.70 \\
9.40\end{array}$ & $\begin{array}{l}23.30 \\
21.75 \\
38.30 \\
33.65 \\
39.65 \\
29.10 \\
44.85 \\
39.00\end{array}$ & $\begin{array}{r}14.50 \\
16.15 \\
22.00 \\
26.05 \\
17.50 \\
15.00 \\
13.30 \\
9.95\end{array}$ & $\begin{array}{r}17.20 \\
16.25 \\
12.90 \\
17.35 \\
9.85 \\
9.95 \\
11.10 \\
10.20\end{array}$ & $\begin{array}{r}9.80 \\
8.25 \\
11.85 \\
12.80 \\
15.70 \\
18.90 \\
16.80 \\
8.20\end{array}$ & $\begin{array}{r}19.90 \\
18.90 \\
13.95 \\
17.35 \\
13.90 \\
9.55 \\
11.15 \\
18.25\end{array}$ & $\begin{array}{l}14.00 \\
13.70 \\
13.15 \\
16.00 \\
18.25 \\
11.10 \\
10.35 \\
12.70\end{array}$ & $\begin{array}{r}8.40 \\
13.45 \\
14.80 \\
16.65 \\
13.90 \\
12.20 \\
18.15 \\
11.60\end{array}$ & $\begin{array}{r}18.20 \\
15.30 \\
18.25 \\
15.85 \\
17.55 \\
10.65 \\
10.40 \\
6.15\end{array}$ & $\begin{array}{l}12.20 \\
15.90 \\
23.90 \\
15.40 \\
12.85 \\
13.70 \\
14.95 \\
12.30\end{array}$ \\
\hline
\end{tabular}


twelve dogs during the four weeks preliminary control period. It is apparent that these data, summarized in Table II, fall within the limits of normal indicated in Table I. Moreover, there were no significant changes in the leukocyte counts of any of the dogs during the nine weeks following operation or during the four weeks following the infections of liver extract. Throughout the experiment, $\operatorname{dog}$ \#, an apparently healthy animal, maintained a leukocyte count considerably higher than the other animals. The reason for this finding is unknown. Figure IV was constructed from the data of Table XI using the arithmetical means of the data of the operated control group and spore injected group. The curves show that there were no significant differences between the averaged leukocyte counts of the two groups of animals during the four weeks preliminary control period; similarly, there were no significant differences between the averaged counts of the two groups during the nine weeks following operation or during the four weeks succeeding liver extract injections. The somewhat higher level of the spore injected group is due to the high counts of dog \#8.

The greylsh-red layer of cells capplng the cellular column of the hematocrit tubes after centrifuging has been mentioned before. This layer of cells, when smeared on a slide and stained, was found to be composed of erythrocytes as well as leukocytes. Since the proportion of red and white cells was not found to be constant, an effort to estimate accurately the 


\section{FIGURE IV}

AVERAGE LEUKOCYTE COUNTS OF EIGHT SPORE INJECTED AND FOUR OPERATED CONTROL DOGS

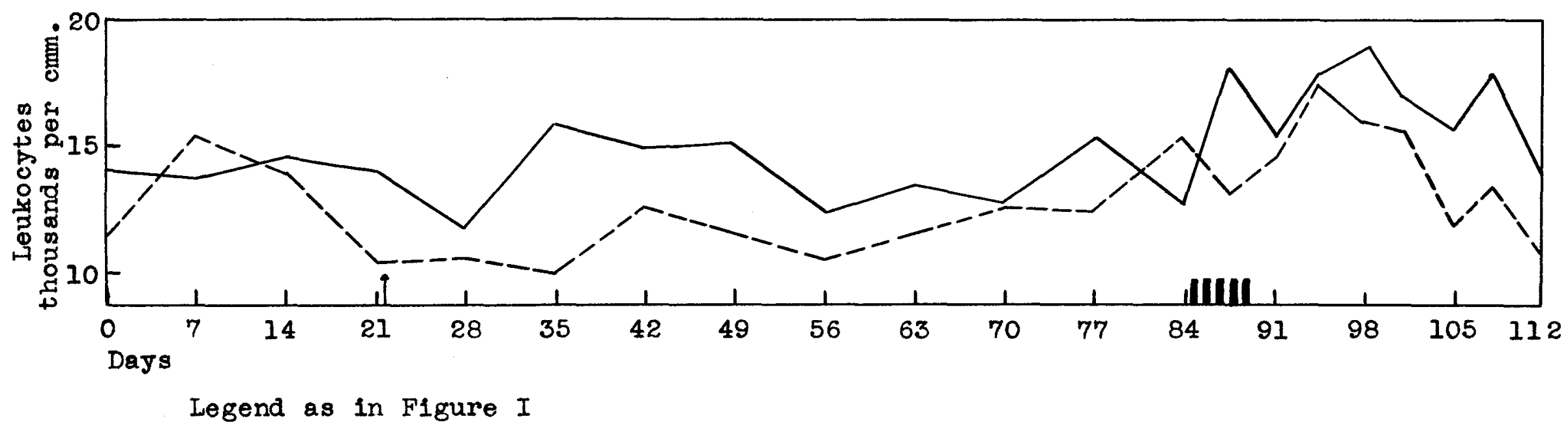


number of leukocytes per volume of blood by the size of the cap was unsuccessful.

Liver function as determined by the Rose Bengal dye excretion test is expressed in per cent according to the colorimetric formula proposed by Delprat and Stowe(37), which is so constructed that $100 \%$ is normal for the normal human I1ver. From Table XII it is seen that during the four weeks preliminary control period values between $77 \%$ and $137 \%$ were obtained. The average of the data for the twelve dogs during this perlod was $107 \%$. Taking these values to represent normal variations, it is evident that there were no significant variations from the above limits during either the nine weeks succeeding operation or the four weeks following the injectIons of liver extract. From Figure V, constructed from the arithmetical means of the data for the operated control and spore injected groups of dogs, it is evident that there were no significant differences between the control and spore injected groups of animals during the four weeks preliminary control period. Further, there were no significant differences between the two groups of animals during the nine weeks followIng operation or the four weeks subsequent to the injections of liver extract.

The blood chemistry studies showed no significant changes in non-protein nitrogen, urea, blood sugar, or $\mathrm{CO}_{2}$ combining power of the plasma in any animal during the entire course of the experiment. The qualitative van den Bergh test 
TABIE XII

ROSE BENGAI TEST RESULTS OF SPORE THJECTED AND OPERATED CONTROI DOGS in per cont

\begin{tabular}{|c|c|c|c|c|c|c|c|c|c|c|c|c|}
\hline \multirow{3}{*}{ Day } & \multicolumn{8}{|c|}{ Spore Injected } & \multicolumn{4}{|c|}{ Operated Control } \\
\hline & \multicolumn{4}{|c|}{ Males } & \multicolumn{4}{|c|}{ Females } & \multicolumn{2}{|c|}{ Mes } & \multicolumn{2}{|c|}{ Females } \\
\hline & 12 & $\$ 4$ & \#6 & $\neq 8$ & H & \#9 & $\$ 10$ & $\$ 12$ & $\# 3$ & $\neq 7$ & f5 & $f 21$ \\
\hline $\begin{array}{r}0 \\
14\end{array}$ & $\begin{array}{r}91 \\
106\end{array}$ & $\begin{array}{l}102 \\
106\end{array}$ & $\begin{array}{l}120 \\
111\end{array}$ & $\begin{array}{l}125 \\
137\end{array}$ & $\begin{array}{r}99 \\
130\end{array}$ & $\begin{array}{l}108 \\
129\end{array}$ & $\begin{array}{l}96 \\
94\end{array}$ & $\begin{array}{l}96 \\
99\end{array}$ & $\begin{array}{l}120 \\
101\end{array}$ & $\begin{array}{l}129 \\
112\end{array}$ & $\begin{array}{r}96 \\
105\end{array}$ & $\begin{array}{r}77 \\
110\end{array}$ \\
\hline 22 & \multicolumn{12}{|c|}{ Operation } \\
\hline $\begin{array}{l}28 \\
42 \\
56 \\
70 \\
84\end{array}$ & $\begin{array}{l}120 \\
116 \\
112 \\
129\end{array}$ & $\begin{array}{l}120 \\
101 \\
121 \\
141\end{array}$ & $\begin{array}{r}91 \\
89 \\
118 \\
133\end{array}$ & $\begin{array}{r}116 \\
126 \\
139 \\
130 \\
92\end{array}$ & $\begin{array}{l}134 \\
123 \\
112 \\
115 \\
109\end{array}$ & $\begin{array}{r}85 \\
109 \\
115 \\
135 \\
133\end{array}$ & $\begin{array}{r}122 \\
117 \\
104 \\
90\end{array}$ & $\begin{array}{l}126 \\
102 \\
133 \\
112\end{array}$ & $\begin{array}{r}117 \\
90 \\
121 \\
128 \\
144\end{array}$ & $\begin{array}{l}113 \\
112 \\
123 \\
101 \\
132\end{array}$ & $\begin{array}{r}124 \\
115 \\
97 \\
97 \\
112\end{array}$ & $\begin{array}{l}107 \\
111 \\
128 \\
119 \\
114\end{array}$ \\
\hline \multicolumn{13}{|c|}{ Iiver Frtract Injected 85th through 89th Days } \\
\hline $\begin{array}{r}98 \\
112\end{array}$ & $\begin{array}{l}117 \\
117\end{array}$ & $\begin{array}{r}134 \\
99\end{array}$ & $\begin{array}{l}116 \\
124\end{array}$ & $\begin{array}{r}127 \\
96\end{array}$ & $\begin{array}{r}122 \\
93\end{array}$ & $\begin{array}{l}113 \\
123\end{array}$ & $\begin{array}{l}113 \\
105\end{array}$ & $\begin{array}{l}136 \\
124\end{array}$ & $\begin{array}{l}116 \\
124\end{array}$ & $\begin{array}{l}76 \\
86\end{array}$ & $\begin{array}{r}91 \\
101\end{array}$ & $\begin{array}{l}125 \\
119\end{array}$ \\
\hline
\end{tabular}




\section{FIGURE V}

AVERAGE ROSE BENGAL LIVER FUNCTION TESTS FOR EIGHT SPORE INJECTED AND FOUR

OPERATED CONTROL DOGS

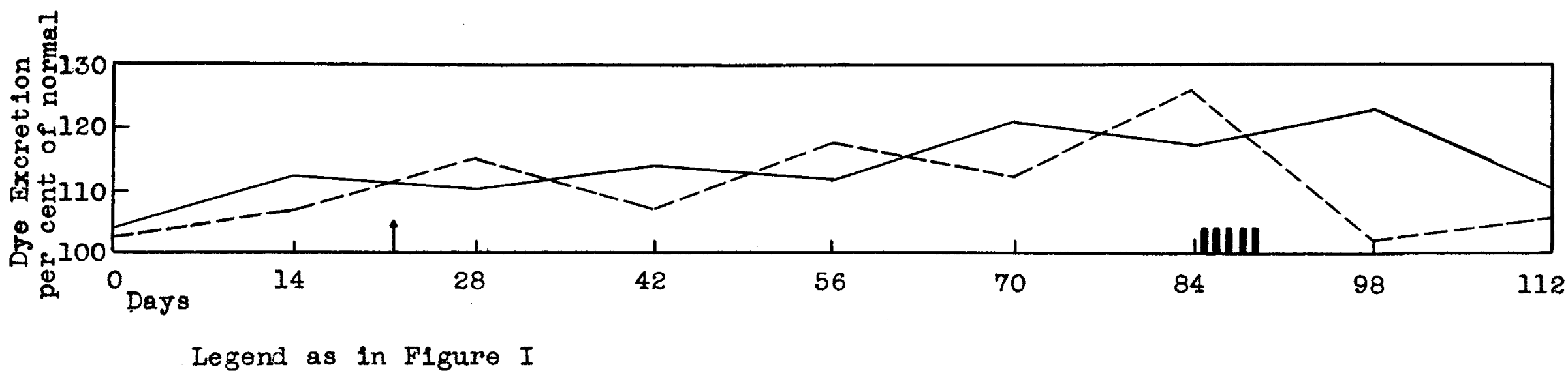


throughout the experiment was recorded as negative on all dogs. Discoloration of the sclerae was never observed in any animals.

Throughout the experiment, the dogs on the whole remained in a state of good health. The abdominal operative wounds closed by primary healing with two exceptions, dogs \#5, and \#II(both of the operated control group); these, however, healed upon inclsion and open drainage. Dog \#IO(spore injected group) developed a keratitis on the eighty-eighth day which subsided with local treatment on the ninety-second day. This animal subsequently went into estrus on the ninetyfifth day. Dog \#I(spore injected group) went into estrus on the one hundred sixth day. During the 112 days of the experiment the spore injected and control animals lost an average of $8.1 \%$ and $8.8 \%$ respectively of their inftial weight.

As previously noted, all the animals except three of the spore injected group were sacrificed and autopsied. The gross findings were entirely unremarkable except for a slight gritty sensation obtained from the knife edge applied to the cut surface of the spore injected livers. The weight of the livers of the spore injected animals was found comparable to the weight of the livers of the control animals: an average of 42 grams per $\mathrm{kg}$. to $40 \mathrm{grams}$ per $\mathrm{kg}$. respectively. There were observed no varices, ulcers or other pathological changes of the gastro-intestinal tract; ascites was not found. The livers of the spore injected animals presented a normal 
gross appearance. The hamstring muscles into which the IIver extract was injected showed no evidence of inflammation (foreign protein reaction), degeneration, or scarring. Sections of the spore infected livers showed that the lobules as a whole were somewhat smaller and stained more Iightly than normal at the center and at the periphery. The normal picture for these dogs was obtained from sections of blopsy material removed at operation. The cells at the center of the lobule which stained lightly were smaller, coarsely granular and contained pycnotic or karyolytic nucle1. The cellular outlines were indistinct and the sinusoids appeared wider due to the shrinking of the liver cords. The cells toward the periphery of the lobule appeared normal. Throughout the section the spores were found imbedded in the portal vessels in a fibrous tissue coat containing hyaline material. These vessels were occluded, but there had developed a collateral circulation composed of one or more thin endothelial tubes which penetrated or skirted the occluding mass. The connective t1ssue of the periportal spaces was increased, but not to the extent of exerting pressure on the newly formed collaterals or the b1le ducts.

\section{DISCUSSION:}

Analysis of the tabulated results discloses variations of the erythrocyte counts together with more or less parallel variations of the hemoglobins and cell concentrations too extensive to be attributed to error due to the methods employed. 
As previously noted, the accuracy of the technique has been verifled in man by wintrobe $(34,35,41)$. In order to exceed errors common to the methods employed, the variations need be of the order of only $2.5 \%$.

The measurements of the mean corpuscular volume, mean corpuscular hemoglobin and mean corpuscular hemoglobin concentration were derived by the same formulae which are applied to the human erythrocyte. These values have been proven accurate and useful $(34,35,42)$. They provide the means by which a macrocytic hyperchromic anemia may be positively identified.

In Table XIII are presented the average erythrocyte, hemoglobin, cell concentration, mean corpuscular volume, mean corpuscular hemoglobin, and mean corpuscular hemoglobin concentration values of normal dogs' blood found in the reports of various investigators together with simflar data from Tables I and II. The reasons for the disagreement between the values listed are not entirely clear. As Wintrobe and his associates $(41,42,47)$ and others $(49,50)$ have pointed out, differences in the method of hemoglobin determination, the type of hematocrit used, and the anticoagulant employed account for some of the lack of closer agreement. A similar situation exists relative to the average blood values of normal men(4I). In my own work, other factors such as the diurnal variation in number(51,52) and in size(53) of the erythrocyte, and in the hemoglobin content of the erythro- 
TABLE XIII

SUMMAFY OF AVERAGE ERYTHROCYTE VAIUES FOR NORMAL DOGS

REPORTED BY DIFFEREAT AUTHORS

\begin{tabular}{|c|c|c|c|c|c|c|}
\hline Author & $\begin{array}{l}\text { Erythrocytes } \\
\text { (millions } \\
\text { per cmm.) }\end{array}$ & $\begin{array}{l}\text { Hemoglobin } \\
\text { (grams } \\
\text { per } 100 \mathrm{cc} \text { ) }\end{array}$ & $\begin{array}{l}\text { Hematocrit } \\
\text { (per cent } \\
\text { cello) }\end{array}$ & $\begin{array}{c}\text { Mean } \\
\text { Corpuscular } \\
\text { Volume } \\
\text { (cubic miera) }\end{array}$ & $\begin{array}{c}\text { Mean } \\
\text { Corpusoular } \\
\text { Homoglobin } \\
\text { (gamma gamma) }\end{array}$ & $\begin{array}{l}\text { Mean } \\
\text { Corpuscular } \\
\text { Hemoglobin } \\
\text { Cone. } \\
\text { (per cent) }\end{array}$ \\
\hline Mayerson (43) & 6.16 & 13.0 & 38.6 & 59.3 & 20.0 & 34.3 \\
\hline mmons (44) & 6.25 & $16.5^{*}$ & 43.0 & 69.0 & $26.4^{*}$ & $38.4^{*}$ \\
\hline $\begin{array}{l}\text { Ashley and } \\
\text { Guest (45) }\end{array}$ & 6.87 & 16.0 & 45.6 & 66.6 & 23.6 & 34.9 \\
\hline $\begin{array}{l}\text { Powers, Bowie } \\
\text { and Howard }(46)\end{array}$ & 7.00 & $16.0^{*}$ & 45.2 & 64.2 & $22.8^{*}$ & $35.3^{*}$ \\
\hline $\begin{array}{l}\text { Wintrobe, } \\
\text { Shumacker, and } \\
\text { Schmidt }(47)\end{array}$ & 7.02 & 14.6 & 47.3 & 67.6 & 21.2 & 31.4 \\
\hline $\begin{array}{l}\text { Leichsenring } \\
\text { et, al.(48) }\end{array}$ & 7.17 & 14.1 & $47.7^{*}$ & $66.4^{*}$ & $19.7^{*}$ & $29.6^{*}$ \\
\hline $\begin{array}{l}\text { Bruner } \\
\text { (Table I) }\end{array}$ & 6.53 & 13.9 & 45.5 & 69.7 & 21.4 & 30.8 \\
\hline $\begin{array}{l}\text { Bruner } \\
\text { (Table II) }\end{array}$ & 6.65 & 14.3 & 46.5 & 69.5 & 21.4 & 30.9 \\
\hline
\end{tabular}

* Calculated from data 
cytes $(52,54)$ have been eliminated as far as possible by the standardized procedure. Varlous other laboratory animals are subject to similar fluctuations and variations of erythrocytes. In Table XJV summarized from Scarborough's compilation(55) are presented briefly these normal varlations. Le1schenring and Honig(56), and Bodansky and Dressler (57) have noted that dogs with high erythrocyte counts tend to have small mean corpuscular volumes. Thus Leischenring and Honig observed a decreased mean corpuscular volume accompanied by a slight polyglobulism following recovery from acute experimental hemorrhage. Bodansky and Dressler state that in their experience, dogs with unusually high erythrocyte counts frequently have small corpuscles. The data obtained from the twelve dogs during the preliminary control period in part confirm these findings in that they show a certain amount of correlation between the number of erythrocytes and the mean corpuscular volume(Table $\mathrm{X}$, Figure III).

The lack of effect of the infection of lycopodium spores on the erythrocytes and hemoglobin shows that this method of liver damage did not produce a quantitative reduction of liver tissue sufficient to cause a deficiency of the ant1-pernicious anemia principle, nor did it interfere significantly in a differential manner with the normal role of the liver in hematopolesis.

Shumacker and Wintrobe(58), found that two of seven 
TABLE XIV

VARIATIONS IN THE ERYTHROCYTE COUNTS

OF NORMAL LABORATORY ANIMALS*

\begin{tabular}{|c|c|c|c|c|}
\hline & $\begin{array}{c}\text { Average } \\
\text { (millions } \\
\text { per cmm.) }\end{array}$ & $\begin{array}{l}\text { Maximum } \\
\text { (millions } \\
\text { per crm.) }\end{array}$ & $\begin{array}{l}\text { Minimum } \\
\text { (millions } \\
\text { per cmm.) }\end{array}$ & $\begin{array}{c}\text { Greatest } \\
\text { range of } \\
\text { variation } \\
\text { found by } \\
\text { one author } \\
\text { (millions } \\
\text { per crm.) }\end{array}$ \\
\hline Rabb1t & 5.62 & 10.0 & 2.8 & 5.5 \\
\hline Guinea pig & 5.75 & 11.8 & 2.5 & 3.6 \\
\hline Rat & 8.50 & 9.9 & 6.4 & 2.6 \\
\hline Mouse & 9.70 & $12 \cdot 4$ & 7.7 & 3.5 \\
\hline DOB & 7.20 & 11.8 & 3.9 & 5.0 \\
\hline Cat & 8.43 & 14.6 & 4.8 & 7.8 \\
\hline Horse & 7.80 & 11.5 & 5.4 & 5.5 \\
\hline $\mathrm{P} 18$ & 6.74 & 10.1 & 3.3 & 6.3 \\
\hline Cattle:Cow & 6.62 & 8.3 & 4.5 & 3.1 \\
\hline $0 x$ & 6.82 & 8.6 & 3.7 & 2.8 \\
\hline Monkey & 5.59 & 7.1 & 3.6 & 3.5 \\
\hline Sheop & 10.38 & 13.0 & 9.0 & 2.4 \\
\hline Goat & 14.42 & 17.0 & 12.2 & 3.8 \\
\hline Chicken & 3.44 & 5.8 & 2.0 & 2.2 \\
\hline Prot & 0.46 & 0.54 & 0.40 & 0.14 \\
\hline
\end{tabular}

Summarized from Scarborough's compllation (55) 
dogs, which were subjected to total gastrectomy and severe cirrhosis of the Ilver produced by carbon tetrachloride, developed a macrocytic hyperchromic anemia shortly before death. This in part suggests that only a small amount of liver tissue is required to maintain a normal blood picture even when the source of the intrinsic factor is reduced. The total amount of the particular liver extract injected into the animals significantly exceeds the amount necessary to induce remission in the average case of pernicious anemia. The liver extract used was a fresh refined concentrate, $3 \mathrm{cc}$. derived from 100 grams of fresh liver. Placed on a per kilogram basis, a $60 \mathrm{~kg}$. pernicious anemia patient in relapse would require less than $0.2 \mathrm{cc}$. per $\mathrm{kg}$. to induce remission. The dogs employed in the present experiment recelved a total of $1.0 \mathrm{cc}$. per $\mathrm{kg}$. over a five day period.

As has been found in this experiment, attempts to accelerate erythropolesis in the normal experimental animal by whole liver or liver extracts have, in general, been unsuccessful. Vaughan and Muller(59) found no effect on the total erythrocytes or the hemoglobin by the addition of whole liver or liver extract to the diet of healthy rats. Landsberg and Thompson(60) failed to find an increased weight or erythrocyte count in guinea pigs after the injection of an active l1ver extract; Jacobson(61), however, has observed 
slight increases in erythrocytes. Adlersburg and Gottsegen (62) have reported a rather unusual finding: working with dogs and rabbits on an ordinary diet they were able to produce a temporary anemia by feeding large doses of German commercial liver preparations effective in pernicious anemia (Hepatrat and Hapatopson).

In the normal human organism, the addition of liver to the diet or the parenteral introduction of liver extracts has been reported as producing slight, if any, effects upon the number of circulating erythrocytes. Cornell(63) has reported no change in the blood of four normal people taking 240 grams of raw Ilver dally for four weeks. Neldhardt and Bannasch(64), feeding raw l1ver to elght persons for perlods varying between seven and thirty days, found only very slight increases in erythrocytes and bemoglobin in seven of elght instances. Jungmann(65) reported practically no changes with the use of a similar diet. Crane, Howard and Murphy(66) have observed slight increases of erythrocytes and hemoglobin upon the ingestion of the powdered extract of 800 grams of I1ver daily for fourteen days. On the other hand, Watkins, Johnson and Berglund(67) observed prompt Increases in erythrocytes amounting to between eight to twenty per cent in the blood of six normal persons during the ingestion of the equivalent of three $\mathrm{kg}$. of liver over a ten day period; every subject developed symptoms and signs of polycythemia 
in varying degrees.

The negative results relative to the erythrocyte counts and hemoglobin values obtalned from the injection of liver extract into the elght spore injected dogs and the four operated control dogs agree, therefore, with the majority of reports on the effect of liver extract on the normal members of different species.

The absence of any significant change in the mean corpuscular volumes and mean corpuscular hemoglobins of the spore injected dogs during the nine weeks following operation, Indicates again that the sequence of normal hematopolesis was not interrupted by the experimental procedure. The effect of the injection of liver extract into normal animals studied from the standpoint of the mean corpuscular volume, mean corpuscular hemoglobin and mean corpuscular hemoglobin concentration has not been reported thus far. It is interesting to note that the liver extract had no effect on the normal sized erythrocytes of either the spore injected or operated control groups. This finding is in agreement with the opinion that the characteristic effect of liver extract on the Price-Jones curve in pernicious anemia $(42,68,69)$ is produced by replacement of the macrocytes with normal sized erythrocytes instead of any direct effect upon the circulating macrocytic erythrocytes.

The average number of reticulocytes, $0.4 \%$, obtained during the preliminary control period compares with values reported by other investigators. Mayerson(43), however, 
found an average reticulocyte count of $1.85 \%$ in thirty-five dogs. He reported an average of $4 \%$ on one dog and found that nine of the thirty-five had average reticulocyte counts higher than the maximal count $(2.8 \%)$ recorded in my own work. Krumbhar (70) found $0.6 \%$ to be the average in the normal dog. Jacobson $(61,71)$ has shown that some hematologically normal guinea pigs respond to the administration of whole Ilver or liver extract by a slight increase in the number of reticulocytes in the circulating blood. The livers of the "reactive" guinea pigs were found to show a reduction in the amount of the stored anti-pernicious anemia principle as established by assay on other "reactive" guinea pigs. It was possible that some or all of the spore infected animals although presenting a normal blood formula, might be in an analagous "reactive" state. The results, however, indicate that there was no significant diminution in the amount of stored ant1-pernicious anemia principle in the livers of the spore injected dogs.

In the norrial human Neidhardt and Bannasch(64), feedIng raw liver, observed that the reticulocyte count rose to a maximum of but 1.2 to 1.7 per cent in seven of elght instances. Crane, Howard, and Murphy(66) have likewise observed negligible changes in reticulocytes upon the ingestIon of a powdered extract of liver. The reticulocyte counts of the spore infected dogs following the injections of liver extract agree with the counts of the operated control dogs, 
and both are comparable with the results obtained in normal humans, indicating further that the erythropoletic systems of the spore injected dogs were not significantly affected by the experimental procedure.

The average leukocyte count of these dogs during the preliminary control period, 13,750 per c. mm., agrees with counts from other sources. Thus, Scarborough(55) found an average of 11,840 , a figure complied from 788 counts by 67 different investigators. He has tabulated in his report average values on normal dogs ranging between 5,700 and 38,000 per c. mm. Mayerson(43) reported 11,165 per c. mm. as the average of 190 counts on 57 dogs with extremes of 5,560 and 19,920. In man, Garrey and his associates $(72,73)$ have observed increases in the leukocyte count following emotion, pain, movement, exercise and active or passive changes in posture. These factors were reduced to a minimum by the standardized procedure of the experiment. The diurnal variation of the leukocytes was controlled by drawing all blood at approximately the same hour of the morning. As already stated, there is no evident explanation for the high counts of dog \#8 during the experiment.

In the case of the dogs whose livers had been injected with spores, there were no indications of the leukocytosis which usually occurs during the process of phagocytosis and removal of autolysing tissue. The unchanged 
leukocyte counts of the spore injected dogs during the period following operation is additional evidence of the absence of a blood plcture resembling perniclous anemia. However, other pathological states accompanied by a macrocytic hyperchromic anemia do not necessarily exhibit a leukopoenia.

Similarly to the lack of significant response in the leukocyte counts to liver administration noted in this experiment, Neldhardt and Bannasch(64) observed no distinct effect on the leukocytes of normal men on a diet of raw Ifver. Watkins, Johnson, and Berglund(67), feeding a liver preparation derived from three $\mathrm{kg}$. of fresh liver during a period of ten days, likewise observed no effect on the leukocytes of six normal men. On the other hand, two groups of investigators $(74,75)$ have reported elevated leukocyte counts within seven hours after a single intramuscular injection of liver extract in man, the count returning to normal on the third day. Their data were obtained from leukocyte counts made at one hour intervals and hence are not suitable for comparison with the leukocyte counts of the spore injected and operated control dogs after the liver extract injections.

It was not surprising that the present study of liver function by means of dye excretion produced negative results. Mann and Bollman(76) investigated the liver function in dogs subjected to surgical removal of $70 \%$ to $90 \%$ of the liver t1ssue. They employed tests of liver function depending on 
the secretion of bile, the relation of the liver to carbohydrate and to protein metabolism, the excretion of dyes, and the detoxifying ability of the liver. With the exception of uric acid metabolism and tests of dye excretion, tests based on these functions produced unreliable results due elther to the activity of other tissues or to the ability of the residual liver tissue, which is necessary for life, to maintain these functions within normal limits. In the case of the dyes, phenoltetrachlorphthalein, bromsulphalein and rose bengal, it was necessary to use relatively large amounts in order to demonstrate definite retention in the blood of dogs whose liver tissue had been reduced to the least amount sustaining life. Of the three dyes used, it appears that rose bengal was the most sensitive to hepatic insufficiency. Using dogs whose livers had been damaged acutely by chemicals, Bollman and Mann(22) have reported that for the most part there was a satisfactory correlation between the degree of injury to the Iiver, as estimated by the gross appearance and histologic section, and the degree of retention of dye(bromsulphalein). However, some of the dogs with extensive cirrhotic changes gave no evidence of dye retention; conversely, a few normal animals with no demonstrable hepatic lesions showed marked dye retention. They also observed that different activities of the liver may be affected to different degrees. Thus, the destruction of uric 
acid is more impaired, than is the formation of urea, by the same degree of liver damage. Here, therefore, is a basis for the possibility that the hematopoletic function of the liver might have been differentially depressed by the procedure employed in this experiment.

Green and Conner(77) Investigated liver function in twelve cases of perniclous anemia by means of the phenoltetrachlorphthalein test, the fructose tolerance test, the serum bilirubin and van den Bergh reaction, and the nitrogen partition of the blood(non-protein nitrogen, urea, uric acid, creatinine, and amino-acid nitrogen). Their positive findings consisted of one instance of a positive direct van den Bergh and serum bilirubin above $5 \mathrm{mgm}$. per cent following a hemolytic crisis, eight instances of serum bilirubin above the limits of normal, and three instances of moderate dye retention. Thus, liver function as tested clinically is not particularly depressed in cases of pernicious anemia in relapse.

It is probable, therefore, that had a macrocytic hyperchromic anemia developed in the spore injected dogs, liver function measured by the Rose Bengal test would not have been depressed.

Mann(78) has found that in the absence of $70 \%$ to $90 \%$ of the liver in dogs there develops a characteristic set of blood chemistry findings consisting of hypoglycemia, a loss 
of formation of urea together with an increase of amino-acid nitrogen, and an increase in uric acid. The absence of such findings in the spore injected animals suggests that at least $30 \%$ of the liver parenchyma was functioning efficiently. It appears that the damage produced by the lycopodium spores was not sufficient to modify the normal role of the liver in carbohydrate and protein metabolism.

Studies of the blood chemistry of pernicious anemia $(77,79,80,81)$ have in general revealed normal or high normal values except for an increased serum bilirubin of 1.4 to 5 mgm. per cent and a "delayed" qualitative van den Bergh test. As already noted above, the spore injected animals continued to show a negative van den Bergh, as did the operated controls. It must be kept in mind, however, that some types of macrocytic hyperchromic anemia are not accompanied by an increased serum bilimbin.

The autopsy findings of the spore injected animals confirmed the evidence pointing to the lack of significant impaiment of liver function. Clinically and experimentally (22) well developed hepatic cirrhosis is accompanied by the development of varices and ascites. The sections of the spore injected livers demonstrated that hepatic cirrhosis did not. develop in spite of occlusion of the intrahepatic portal radicles by the spores. An intrahepatic collateral circulation such as has been described has not been previously reported so far as is known. About three-fourths of the liver cells 
seen in the autopsy sections of the spore injected dogs were histologically normal.

The liver in pernicious anemia(6) grossly is a pale rusty color and not obviously enlarged. The cut surface is dry and shows musty brown lobules with yellow centers. Sections show that the brown in the periphery of the lobule is due to the deposition of iron; the pale centers show fatty degeneration.

The experimental findings in this attempt to produce a macrocytic hyperchromic anemia by the intraportal injection of lycopodium spores in the dog show that the damage which can be produced by this method is insufficient to affect measurably the physiologic storage activity of the liver for the antipernicious anemia principle. The fallure of the method probably is due to the reserve of 11 ver function and to the regenerative properties of Iiver tissue.

of the three spore injected dogs saved for future examination, one died on the 208th day after the injection of lycopodium spores from an unknown cause before counts were made. The other two showed normal blood pictures when examined 271 and 334 days after the injection of Iycopodium spores.

\section{SUMMARY:}

1. The average values and range of variation for erythrocytes, hemoglobin, per cent packed cells, mean corpuscular volume, mean corpuscular hemoglobin, mean corpuscular hemoglobin concentration, reticulocytes, and 
leukocytes from 63 counts on 19 normal dogs and from 48 counts on 12 normal dogs are given. Variations definitely in excess of the technical error of $2.5 \%$ are normally found in counts seven days apart.

2. Lycopodium spores in a $1 \%$ suspension in phys10logical sodium chloride solution were introduced into the intrahepatic radicles of the portal vein of four male and four female dogs with the hope of injuring the function of the liver in hematopolesis sufficiently to produce a macrocytic hyperchromic anemia. Two male and two female dogs were similarly infected with physiological sodium chloride solution to serve as operated control animals.

3. The elght spore injected dogs throughout a period of nine weeks gave no evidence of the development of any type of anemia as seen by the unchanged erythrocyte counts, hemoglobin values, per cents of packed cells, mean corpuscular volumes, mean corpuscular hemoglobins, mean corpuscular hemoglobin concentrations and leukocyte counts. Further, there was no evidence of damage to other functions of the liver as studied by the Rose Bengal dye excretion test and blood chemistry determinations.

4. Liver extract in clinically adequate amounts produced no change in the blood constituents studied, including the reticulocytes, in either the spore injected or operated control groups of dogs. 
5. Autopsy examination of the spore injected dogs showed essentially normal gross findings. Microscopic study of sections of the livers of the spore injected dogs showed that the Iycopodium spores were embedded in hyaline fibrous tissue in the portal spaces, and that fully three-fourths of the liver tissue was histologically normal.

6. This attempt at the production of a macrocytic hyperchromic anemia in the dog, therefore, was unsuccessful. 


\section{BIBLIOGRAPHY:}

(1) Castle, W. B., and Townsend, W. C.: Observations on the Et lological Relationship of Achylla Gastrica to Pernicious Anemia; The Effect of Administration to Patients with Pernicious Anemia of Beef Muscle after Incubation with Normal Human Gastric Juice, Am. J. M. Sc. 178: 764, 1929 .

(2) Wilkinson, J. F., and Klein, L.: Relationship between Ant1-Anaemic Principles in Stomach and Liver, Lancet 2: $629,1933$.

(3) Castle, W. B.; Townsend, W. C., and Heath, C. W.: Further Observations on the Aetiological Relationship of Achylia Gastrica to Pernicious Anaemia, Lancet 1: $1062,1930$.

(4) Castle, W. B.; Heath, C. W., and Strauss, H. B.: Observations on the Etiologic Relationship of Achylia Gastrica to Pernicious Anemia; Blologic Assay of Gastric Secretions of Patients with Pernicious Anemia Having Free Hydrochloric Acid and that of Patients without Anemia Having No Free Hydrochloric Acid, and of the Role of Intestinal Impermeability to Hematopoletic Substance in Pernicious Anemia, Am. J. M. Sc. 182: 741, 1931.

(5) Meulengracht, E.: Pernicious Anemia in Intestinal Stricture (with One Liver Treated Case), Acta Med. Scandinav. 72: 231, 1929.

(6) Vaughan, J.: The Anaemias, London, Oxford University Press, 1934.

(7) Seyderhelm, R.: Die Bedeutung des Dunndarm fur die Genese der Perniziosen Anamie, Klin. Wchnschr. 3: 568, 1924.

(8) Meulengracht, E.: Dunndarm Strikturen und Perniziose Anamie Darmresection. Elnige Bemerkungen uber die Darmflora, Acta Med. Scandinav. 56: 432, 1922 .

(9) Klein, L., and Wilkinson, J. F.: Investigation on the Nature of Haemopolet in, the Ant1-Anaemic Substance in Hog's Stomach; The Production of a Thermostabile Haemopoietically Active Substance Similar to or Identical with The Anti-Anaemic Principle of Liver by the Action of Thermolabile Haemopolet in on Beef, Biochem. J. 28: 1684, 1934. 
(10) Richter, 0.; Ivy, A. C., and Kim, M. S.: Action of Human "Perniclous Anemia Liver Extract," Proc. Soc. Exper. Blol. \& Med. 29: 1093, 1932.

(11) Goldhamer, S. M.: Liver Extract Therapy in Cirrhosis of the Liver; Relation of Ifver Dysfunction to Nonstorage of "Ant1-Anemic" Substances in Producing a Blood Picture Resembling Pernicious Anemia in a Patient Secreting Free Hydrochloric Acid, Arch. Int. Med. 53: 54, 1934 .

(12) Goldhamer, S. M.; Isaacs, R., and Sturgis, C. C.: Role of the Liver in Hematopolesis, Am. J. M. Sc. 188: $193,1934$.

(13) Wilkinson, J. F., and Klein, L.: Haematopoietic Activity of Normal and Abnormal Human Livers with Special Reference to Pernicious Anaemia, Quart. J. Med. 3: $341,1934$.

(14) Gamna, C.: Uber Veranderungen der roten Blutkorperchen be1 Lebererkrankungen, XIIn. Wchnschr. 12: $348,1933$.

(15) Holler, G., and Kudelka, O.: Resultate von Bestimmungen des Erythrocytendurschmessers beim Menschen unter Physiologischen und Pathologischen Verhaltnissen. III Mitteilungen. Das Verhalten des Durchmessers der Erythrocyten be1 Erkrankungen der Bauchspeicheldruse, Deutsches Arch. f. klin. Med. 154: 172, 1926.

(16) Wintrobe, M. M., and Shumacker, H. S., Jr.: Occurrence of Macrocytic Anemia in Association with Disorders of the Liver, together with Consideration of the Relation of this Anemia to Perniclous Anemia, Bull. Johns Hopkins Hosp. 52: 387, 1933.

(17) Van Duyn, J., Jr.: Macrocyt1c Anemia in Diseases of the Iiver, Arch. Int. Med. 52: 839, 1933.

(18) Malamos, B.: Das rote Blutb1ld bel Lebererkrankungen, Deutsches Arch. f. klin. Med. 177: 209, 1935.

(19) Sa1to, H.: The Experimental Study on the Influence of the Excision of Liver on the Constituents of the Blood, Se1-I-Kwa1 M. J. 37: 1, 1918.

(20) Bracaloni, E., and Montonar1, A.: The Morphological Picture of the Blood of Rabbits Operated by Subtotal Hepatectomy, R1v. d1. clin. med. 33: 897, 1932. 
(2I) Pelligrin1, G.: Changes in the Blood and Hematopoletic Organs after Liver Excision in the Frog, Haematologica, I. Arch. 13: 85, 1932.

(22) Bollman, J. L., and Mann, F. C.: Experimentally Produced Lesions of the Liver, Ann. Int. Med. 5: 699, 1931.

(23) Mann, F. C.: Personal Communication to Dr. G. E. Wakerlin, 1934.

(24) Higgins, G. M., and Stasney, J.: Macrocytic Anemia in Experimental Cirrhosis, Proc. Staff heet. Mayo Clin. 10: 429, 1935 .

(25) Richter, 0.; Ivy, S. C., and Meyer, A. F.: Study of Dog's Stomach and Liver for Substances Effective in Pernicious Anemia, Proc. Soc. Exper. Blol. \& Med. 31: 550,1934 .

(26) Strauss, M. B., and Castle, W. B.: Amount of Material. Effective in Pernicious Anemia Present in Dog Liver, Proc. Soc. Exper. Blol. \& Med. 31: 360, 1933.

(27) Storte, E.: Experimental Bothriocephalus Anemia, Haematologica, I. Arch. 12: 237, 1931.

(28) Seyderhelm, R.; Lehmann, W., and Wichels, P.: Experimentalle Intestinale Perniziose Anamie beim Hund, Klin. Wchnschr. 3: 1439, 1924.

(29) Mall, F. P.: A Study of the Structural Unit of the Liver, Am. J. Anat. 5: 227, 1906.

(30) Da Costa, J. C.: Principles of Surgery, ed. 7, Philadelphia, W. V. Saunders Co., 1914.

(31) Elman, R., and Cole, W. H.: Loss of Blood as a Factor In Death from Acute Portal Obstruction, Proc. Soc. Exper. Biol. \& Hed. 29: 1122, 1932.

(32) Scott, H. G., and Wangensteen, O. H.: Blood Losses in Experimental Intestinal Strangulations and Their Relationship to Degree of Shock and Death, Proc. Soc. Exper. Biol. \& Med. 29: 748, 1932.

(33) Simonds, J. P., and Brandes, W. W.: The Effect of Obstruction of the Hepatic Veins on the Systemic Circulation, Am. J. Physiol. 72: 320, 1925. 
(34) Wintrobe, M. M.: The Size and Hemoglobin Content of the Erythrocyte; Hethods of Determination and Clinical Application, J. Lab. \& Clin. Med. 17: 899, 1932.

(35) Wintrobe, M. M.: Anemia; Classification and Treatment on the Basis of Differences in Average Volume and Hemoglobin Content of Red Corpuscles, Arch. Int. hed. 54: 256, 1936 .

(36) Stitt, E. R.: Practical Bacteriology, Blood Work and Animal Parasitology, ed. 8, Philadelphia, P. Blak1ston's Son \& Co., 1927 .

(37) Delprat, G. D., and Stowe, W. P.: The Rose Bengal Test for Liver Function; Historical Sketch and Improved Technique, J. Lab. \& Clin. Med. 16: 923, 1931.

(38) Delprat, G. D.: Studies on Iiver Function; Rose Bengal Elimination from the Blood as Influenced by Liver Injury, Arch. Int. Med. 32: 401, 1923.

(39) Kikuth, W.: Die Bartonellen, elne neue Gruppe von Anamieerregern, Wunchen. Med. Wchnschr. 75: 1595, 1928.

(40) Perard, C.: Infection du chien par Bartonella canis, Compt. rend. Soc. de B101. 100: 1111, 1929.

(4I) Wintrobe, M. M., and Miller, M. H.: Normal Blood Determinations in the South, Arch. Int. Med. $43: 96,1929$.

(42) Wintrobe, M. M.: The Hemoglobin Content, Volume, and Th1ckness of the Red Blood Corpuscle in Pernicious Anemia and Sprue, and Changes Associated with Liver Therapy, Am. J. Med. Sc. 181: 217, 1931.

(43) Mayerson, H. S.: The Blood Cytology of Dogs, Anat. Rec. 47: 239,1930 .

(44) Emmons, W. F.: The Interrelation of Number, Volume, Diameter and Area of Mammalian Erythrocytes, J. Physiol. 64: 215, 1927.

(45) Ashley, A., and Guest, C. M.: The Distribution of Blood Phosphorous after Suppression of Renal Function, J. Clin. Investigation 13: 219, 1934 . 
(46) Powers, J. H.; Bowle, M. A., and Howard, I. M.: Some Observations on the Blood of Normal Dogs, with Special Reference to the Total Volume, Am. J. Phys101. 92: 665, 1930.

(47) Wintrobe, M. M.; Shumacker, H. B., and Schmidt, W. J.: Values for Number, Size, and Hemoglobin Content of Erythrocytes in Normal Dogs, Rabbits, and Rats, Am. J. Physiol. 114: 502, 1936.

(48) Lelchsenring, J. M.; Blester, A.; Honig, H. H.; Furnas, S. M.; Foss, E. S., and Routt, M. V.: Blood Regeneration Studies; Observations on the Blood of Normal Dogs with Special Reference to the Measurement of Volume, Erythrocytes, Leucocytes and Nitrogenous Constituents, Am. J. Phys101. 99: 391, 1931.

(49) Guest, G. M., and Siler, V. E.: A Centrifuge Method for the Determination of Volume of Cells in Blood, J. Lab. \& Clin. Med. 19: 757, 1934.

(50) Heller, V. G., and Paul, H.: Changes in Cell Volume Produced by Varying Concentrations of Different Ant1coagulants, J. Lab. \& Clin. Med. 19: 777, 1934.

(51) Smith, C.: Normal Variations in Erythrocyte and Hemoglobin Values in Women, Arch. Int. Ned. 47: 206, 1931 .

(52) Short, J. J.: Diurnal Variations in Concentration of Red Blood Cells and Hemoglobin, J. Lab. \& Clin. Med. 20: 708, 1935.

(53) Price-Jones, C.: The Diurnal Variation in sizes of the Red Blood Cells, J. Path. \& Bact. 23: 371, 1920.

(54) Rabinowitch, I. M.: Variations of Percentage of Hemoglobin in Man During the Day, J. Lab. \& Clin. Med. 9: $120,1923$.

(55) Scarborough, R. A.: The Blood Picture of Normal Laboratory Animals, Yale J. B10l. \& Med. 3: 359, 1930. Iblá $3: 63 ; 169 ; 267 ; 431 ; 547,1930$. Ibld $4: 69 ; 199 ; 323 ; 740,1931$.

(56) Leichsenring, J. M., and Honig, H. H.: Blood Regeneration Studies; Changes in Volume, Number and Size of Erythrocytes in Hemorrhagic Anemia, Am. J. Physiol. 98: 636,1931 . 
(57) Bodansky, M., and Dressler, O. G.: Distribution of Water and Cholesterol in the Blood in Experimental Anaemia, Quart. J. Exper. Phys101. 17: 157, 1927.

(58) Shumacker, H. B., and Wintrobe, M. M.: Experimental Gastrectomy; Effects on the Blood Marphology, Especially when Complicated by Infection or Iiver Damage, Bull. Johns Hopkins Hosp. 57: 384, 1936.

(59) Vaughan, J. K., and Muller, G. L.: The Effect of Liver and Commercial Liver Extract on the Body Weight, Red Blood Cells, and Reticulocytes of Normal Rats, J. Clin. Investigation 11: 129, 1932.

(60) Landsberg, J. W., and Thompson, M. R.: The Guinea Pig as a Hematopoletic Test Animal; Preliminary Report, J. Am. Pharm. A. 23: 964, 1934 .

(61) Jacobson, B. M.: The Response of the Guinea Pig's Reticulocytes to Substances Effective in Pernicious Anemia; A Biologic Assay of the Therapeutic Potency of Liver Extracts, J. Clin. Investigation 14: 665, 1935.

(62) Adlersberg, D., and Gottsegen, G.: Wirkung der Leberextrakt in Tierversuch, Arch. f. exper. Path. u. Pharmakol. 142: 323, 1929.

(63) Cornell, B. S.: The Effects of Liver in the Diet on the Blood of Normal Persons, Canad. M. A. J. 18: 394,1928 .

(64) Neidhardt, K., and Bannasch, K.: Zur Kenntnis des Einflusses der Leberdiat auf den gesunden Organismus, Ztschr. f. klin. Med. 11l: 292, 1929.

(65) Jungmann, $P_{\text {.: }}$ Uber die Wirkungsweise der Leberdiat be1 der perniziosen Anamie, Klin. Tchnschr. 7: 44I, 1928.

(66) Crane, M. P.; Howard, I., and Murphy, W. P.: The Effect of I1ver Extract on the Blood of Normal Persons, Am. J. M. Sc. 180: 803, 1930 .

(67) Watkins, C. H.; Johnson, R., and Berglund, H.: Effect of Liver Extract on Erythrocytes and Reticulocytes in Normal Individuals, Proc. Soc. Exper. B1ol. \& Med. 25: 720, 1928. 
(68) Price-Jones, C.: Red Cell Diameters in 100 Healthy Persons and in Pernicious Anemia; Effect of I1ver Treatment, J. Path. \& Bact. 32: 479, 1929.

(69) Fitzhugh, G., and Persons, E. L.: Studies on Red Blood Cell Diameter; The Decrease in the Mean Diameter of the Reticulocytes and Adult Red Blood Cells in Pernicious Anemia Following Liver Therapy, J. Clin. Investigation 7: 631,1929 .

(70) Krumbhaar, E. B.: Reticulosis - Increased Percentage of Reticulated Erythrocytes in Peripheral Blood, J. Lab. \& Clin. Med. 8: 11, 1922.

(71) Jacobson, B. M.: On a Pernicious Anemia-like State in the Guinea P1g, J. Clin. Investigation 13: 714, 1934.

(72) Garrey, W. E., and Butler, V.: Physlological Leucocytosis, Am. J. Phys101. 90: 355, 1929.

(73) Garrey, W. E., and Bryan, W. R.: Varlations in White Blood Cell Counts, Physiol. Rev. 15: 597, 1935.

(74) Powers, J.H., and Murphy, W. P.: Leucocytosis Following Intramuscular Injection of Liver Extract, J. Clin. Investigation 12: $713,1933$.

(75) Meyer, 0. O.; Middleton, W. S., and Thewlis, E. M.: Leukocytosis after Parenteral Injection of Liver Extract, Am. J. M. Sc. 188: 49, 1934 .

(76) Mann, F. C., and Bollman, J. L.: Liver Function Tests, Arch. Path. \& Lab. Med. I: 681, 1926.

(77) Greene, C. H., and Conner, H. M.: Diseases of the Liver; A Comparative Study of the Tests for Hepatic Function in Certain Diseases of the Hematopoietic System, Arch. Int. Med. 38: 167, 1926.

(78) Mann, F. C.: The Effects of Complete and Partial Removal of the Liver, Medicine 6: 419, 1927.

(79) Kahn, M., and Barsky, J.: Studies on the Chemistry of Pernicious Anemia, Arch. Int. Med. 23: 334, 1919.

(80) Gettler, A. O., and Lindeman, E.: The Blood Chemistry of Pernicious Anemia, Arch. Int. Med. 26: 453, 1920.

(81) Brown, G. 0.; Ames, 0.; Warren, S., and Peabody, F. W.: Blood Pigments in Pernicious Anemia, J. Clin. Investigation $1: 295,1925$. 